\title{
Influenza A virus-induced thymus atrophy differentially affects dynamics of conventional and regulatory $\mathrm{T}$ cell development
}

\author{
Yassin Elfaki ${ }^{1, *}$, Philippe A. Robert ${ }^{2,}$, Christoph Binz ${ }^{3}$, Christine S. $\underline{\text { Falk }}^{4}$, Dunja Bruder $^{5,6}$, \\ Immo $\underline{\text { Prinz }}^{3,7}$, Stefan $\underline{\text { Floess }}^{1}$, Michael Meyer-Hermann ${ }^{2,7,8}$ and Jochen $\underline{\text { Huehn }}^{1,7 \dagger}$ \\ ${ }^{1}$ Department of Experimental Immunology, Helmholtz Centre for Infection Research, Braunschweig, Germany \\ ${ }^{2}$ Department of Systems Immunology and Braunschweig Integrated Centre of Systems Biology, Helmholtz Centre for \\ Infection Research, Braunschweig, Germany \\ 3Institute of Immunology, Hannover Medical School, Hannover, Germany \\ ${ }^{4}$ Institute of Transplant Immunology, Hannover Medical School, Hannover, Germany \\ 5Infection Immunology Group, Institute of Medical Microbiology, Infection Control and Prevention, Health Campus \\ Immunology, Infectiology and Inflammation, Otto-von-Guericke University Magdeburg, Magdeburg, Germany \\ ${ }^{6}$ Immune Regulation Group, Helmholtz Centre for Infection Research, Braunschweig, Germany \\ ${ }^{7}$ Cluster of Excellence RESIST (EXC 2155), Hannover Medical School, Hannover, Germany \\ ${ }^{8}$ Institute for Biochemistry, Biotechnology and Bioinformatics, Technical University Braunschweig, Braunschweig, Germany
}

*The author contributed equally to this work.

${ }^{\dagger}$ correspondence to: Jochen Huehn, Department of Experimental Immunology, Helmholtz Centre for Infection Research, Inhoffenstr. 7, 38124 Braunschweig, Germany; phone: +49 5316181 3310; e-mail: jochen.huehn@helmholtz-hzi.de

Key words: thymus; Foxp3+ Treg cells; thymus atrophy; influenza A virus; mathematical modeling; ordinary differential equations

\footnotetext{
Abbreviations: AICc, corrected Akaike Information Criterion; DP, double-positive; dpi, days post infection; IAV, influenza A virus; IDP, late double-positive; ODE, ordinary differential equation, SP, single-positive; Tconv, conventional T cell; TCR, T cell receptor; Treg, regulatory $\mathrm{T}$ cell
} 


\section{ABSTRACT}

Foxp3 ${ }^{+}$regulatory $\mathrm{T}$ (Treg) cells, which are crucial for maintenance of self-tolerance, mainly develop within the thymus, where they arise from CD25+Foxp3- or CD25-Foxp3 ${ }^{+}$Treg cell precursors. Although it is known that infections can cause transient thymic involution, the impact of infection-induced thymus atrophy on thymic Treg (tTreg) cell development is unknown. Here, we infected mice with influenza A virus (IAV) and studied thymocyte population dynamics post infection. IAV infection caused a massive, but transient thymic involution, dominated by a loss of $\mathrm{CD} 4^{+} \mathrm{CD} 8^{+}$double-positive (DP) thymocytes, which was accompanied by a significant increase in the frequency of CD25+Foxp $3^{+}$tTreg cells. Differential apoptosis susceptibility could be experimentally excluded as a reason for the relative tTreg cell increase, and mathematical modeling suggested that enhanced tTreg cell generation cannot explain the increased frequency of tTreg cells. Yet, an increased death of DP thymocytes and augmented exit of single-positive (SP) thymocytes was suggested to be causative. Interestingly, IAV-induced thymus atrophy resulted in a significantly reduced T cell receptor (TCR) repertoire diversity of newly produced tTreg cells. Taken together, IAVinduced thymus atrophy is substantially altering the dynamics of major thymocyte populations, finally resulting in a relative increase of tTreg cells with an altered TCR repertoire. 


\section{INTRODUCTION}

$\mathrm{CD}^{+}$regulatory $\mathrm{T}$ (Treg) cells, which express the lineage-specification factor forkhead box P3 (Foxp3), constitute a subset of CD4 ${ }^{+} \mathrm{T}$ cells that is crucial for the maintenance of immune homeostasis and self-tolerance [1]. The majority of the Treg cell population (approximately $80 \%$ ) originates from the thymus, and hence these cells are termed thymus-derived Treg (tTreg) cells [2]. In the thymus, the development of tTreg cells proceeds through a two-step process involving $\mathrm{T}$ cell receptor (TCR) and cytokine signaling that first produces CD25+Foxp3- or CD25-Foxp3 ${ }^{+}$Treg cell precursors, which in a second step contribute almost equally to the generation of mature CD25+Foxp3 ${ }^{+}$tTreg cells [3-6].

Thymus atrophy is a condition whereby the size and function of the thymus are greatly reduced. Many stimuli have been shown to result in thymus atrophy, including stress, inflammation, infections, malnutrition, pregnancy, and aging [7-12]. The evolutionary benefit of thymus atrophy is still debated, with both metabolic and immunologic hypotheses being proposed. For instance, infection-induced thymus atrophy is thought to be beneficial, since a temporary cessation in T cell production might limit the effect of dominant tolerance on the course of the infection [13]. Interferon- $\alpha$ has been reported as a critical molecular mediator of infection-induced thymus atrophy, and sensitivity of the thymic epithelium is controlled by a sophisticated microRNA network $[14,15]$. It is important to note that thymic involution during infection is a very rapid yet transient phenomenon, with recovery within one to two weeks [14]. However, despite this ample knowledge it has not yet been elucidated whether infection-induced thymus atrophy leads to changes in tTreg cell development.

Mathematical modeling has been used to describe the dynamics of thymocyte populations at steady state [16] and following drug-induced atrophy [17, 18], or to unravel determinants of the CD4:CD8 ratio in which T cells are generated by thymopoiesis [19]. However, these models did not consider mature tTreg cells and their precursors, and a new model is needed to understand tTreg cell dynamics during infection-induced atrophy.

Influenza A virus (IAV) is a single-stranded RNA virus belonging to Orthomyxoviridae family of viruses. It is an important respiratory pathogen that caused many epidemics and pandemics [20]. IAV has been shown to induce thymus atrophy accompanied by lymphopenia, and this IAV-induced thymus atrophy is mainly characterized by loss of $\mathrm{CD}^{+} \mathrm{CD}^{+}$double-positive (DP) thymocytes [21]. Several mechanisms have been suggested to contribute to IAV-induced thymus atrophy, including direct interference with 
T cell development, elevated IFNy production by innate CD8 $\alpha^{+}$T cells and/or NK cells in the thymus, and increased levels of glucocorticoids [21-23]. Yet, consequences of IAV-induced thymus atrophy on tTreg cell development are unknown.

In the present study, we have investigated the dynamics of major thymocyte populations during IAV-induced thymus atrophy. The infection-induced thymic involution was dominated by a rapid yet transient loss of DP thymocytes and a simultaneous significant increase in the frequency of newly generated CD25-Foxp $3^{+}$precursors and CD25+Foxp $3^{+}$tTreg cells, the latter interestingly showing a significantly reduced TCR repertoire diversity. When studying the molecular mechanisms underlying this phenomenon, mathematical modeling suggested an involvement of an increased death of DP thymocytes and an augmented exit of SP thymocytes from the thymus to be causative for the relative increase in newly generated CD25+Foxp $3^{+}$tTreg cells at the peak of IAV-induced thymus atrophy.

\section{RESULTS}

\section{Frequency of tTreg cells and their CD25-Foxp $3^{+}$precursors increases upon IAV- induced thymus atrophy}

IAV infection has been reported to cause a transient thymic involution [21, 23]. In order to study the impact of IAV-induced thymus atrophy on tTreg cell development, we performed a kinetic analysis by infecting adult Foxp ${ }^{\mathrm{hCD} 2} \times \mathrm{Rag} 1^{\mathrm{GFP}}$ double reporter mice with a mouseadapted strain of IAV (PR8M) and analyzing their thymi at different time points post infection. Under the experimental conditions chosen, thymus atrophy was evident most dramatically 10 days post infection (dpi), both macroscopically and regarding total cellularity (Fig. 1A). In line with previously published data [23], this drop was attributable to a loss of DP thymocytes (Fig. 1B, Supporting Information Fig. 1 and 'Supporting Information Mathematical Model'). Surprisingly, coinciding with the peak of atrophy, the frequency of CD25+Foxp $3^{\text {hCD2+ }}$ Treg cells among CD4 single-positive (SP) thymocytes increased significantly in thymi from IAVinfected mice when compared to PBS-treated controls (Fig. 1C and Supporting Information Fig. 1). Furthermore, a modest, though not significant, increase in the frequency of CD25-Foxp $3^{\text {hCD2+ }}$, but not CD25+Foxp3 ${ }^{\text {hCD2- }}$ Treg cell precursors was observed (Fig. $1 \mathrm{C}$ and Supporting Information Fig. 1). Taken together, these data show that IAV-induced thymus atrophy results in an increase in the frequency of tTreg cells and their CD25-Foxp3 ${ }^{+}$ precursors. 
A
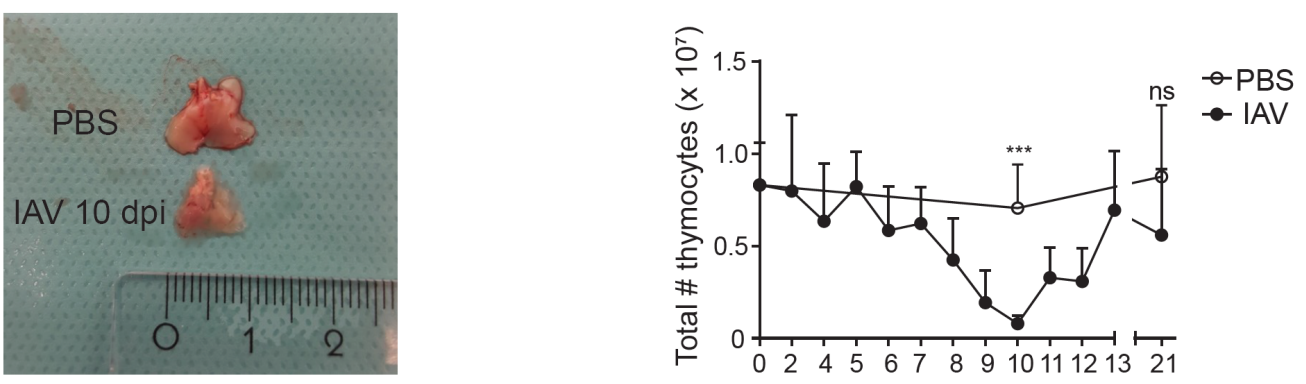

B
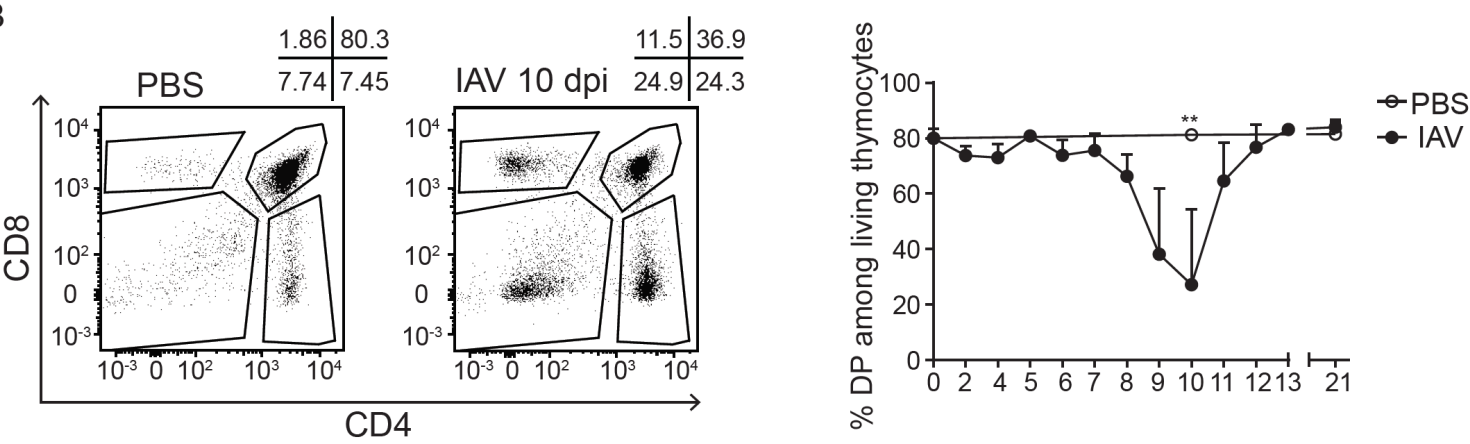

c
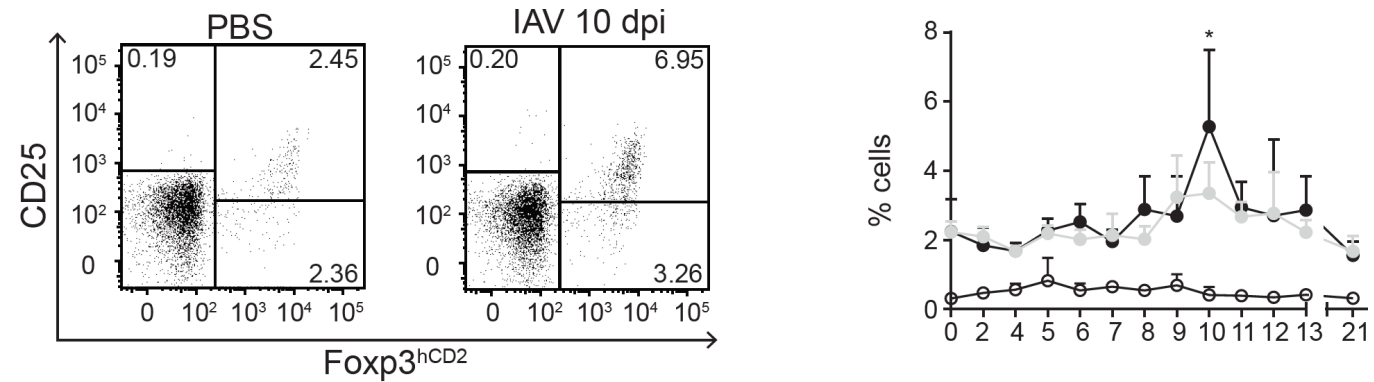

$\rightarrow \mathrm{CD}^{+} 5^{+} \mathrm{Foxp} 3^{\mathrm{hCD} 2+}$ CD25-Foxp3 3 hD2+ $\rightarrow$ CD25+Foxp3 ${ }^{\text {hCD2- }}$

Figure 1. Frequency of tTreg cells and their CD25-Foxp $3^{+}$precursors increases upon IAV-induced thymus atrophy. Foxp $3^{\mathrm{hCD} 2} \times$ Rag $1^{\mathrm{GFP}}$ double reporter mice were infected with IAV and control mice received PBS. Thymi were analyzed at different days post infection (dpi). (A) (Left) Thymic morphology and gross size of PBS-treated and IAV-infected mice at $10 \mathrm{dpi}$. Representative of 9-20 animals per group. (Right) Graph summarizes thymic cellularity collected from PBS-treated (open circles) and IAV-infected (filled circles) mice at indicated dpi. (B) (Left) Representative dotplots depict gating strategy to identify CD4SP, CD8SP, DP and DN populations among living thymocytes prepared from PBS-treated and IAV-infected mice at $10 \mathrm{dpi}$. Numbers indicate frequencies of cells in the respective indicated gates. (Right) Graph summarizes frequency of DP thymocytes among living thymocytes prepared from PBS-treated (open circles) and IAV-infected (filled circles) mice at the indicated dpi. (C) (Left) Representative dotplots depict gating strategy to identify CD25 $5^{+}$Foxp3 ${ }^{\text {hCD2+ }}$ Treg cells, CD25-Foxp3 ${ }^{\text {hCD2+ }}$ Treg cell precursors and CD25+Foxp3 ${ }^{\text {hCD2- }}$ Treg cell precursors among CD4SP thymocytes from PBS-treated and IAV-infected mice at $10 \mathrm{dpi}$. Numbers indicate frequencies of cells in indicated gates. (Right) Graph summarizes frequencies of CD25+Foxp3 ${ }^{\text {hCD2+ }}$ Treg cells (filled circles) and their precursors (CD25+Foxp3 ${ }^{\text {hCD2- }}$ (open circles) and CD25-Foxp3 ${ }^{\text {hCD2+ }}$ (grey circles)) among CD4SP thymocytes at the indicated dpi. Data were pooled from at least two independent experiments with 5-9 mice per group and presented as mean + SD. Each dot represents mean of biological replicates. Mann-Whitney test was used to test for statistical significance and significance was calculated by comparing CD25+Foxp3 ${ }^{\text {hCD2+ }}$ Treg cells, CD25-Foxp $3^{\text {hCD2+ }}$ Treg cell precursors and CD25+Foxp3 ${ }^{\text {hCD2- }}$ Treg cell precursors from IAV-infected mice at $10 \mathrm{dpi}$ and PBS-treated controls (not depicted). 


\section{IAV infection results in increased frequency of newly generated tTreg cells}

Treg cells in the thymus are a diverse population, consisting of newly generated bona fide tTreg cells as well as recirculating Treg cells, which re-enter the thymus from the periphery [24-29]. We next asked if the abovementioned overrepresentation of Treg cells within atrophied thymi maps to the newly generated or recirculating Treg cells, or both. Hence, we here made use of transgenic Rag1 ${ }^{\text {GFP }}$ reporter mice, which express green fluorescent protein (GFP) under control of the recombination-activating gene 1 (Rag1) promoter [30]. In these mice, GFP expression identifies newly generated thymocytes and discriminates them from older and/or re-circulating $T$ cells. In line with previously published data [24], the vast majority of CD4SP thymocytes was newly generated (Rag1 ${ }^{\text {GFP+ }}$ ) (Fig. 2A and Supporting Information Fig. 1). Interestingly, IAV-infected mice at 10 dpi showed a significant increase

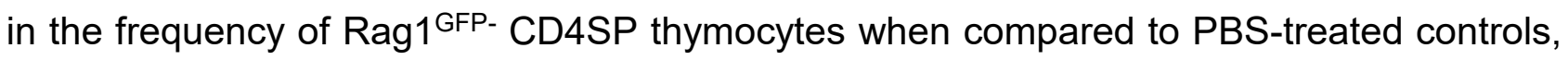
yet their absolute numbers remained unchanged (Fig. 2A and Supporting Information Fig. 2A).

Among newly generated Rag $1^{\mathrm{GFP}+}$ CD4SP thymocytes, we observed a significant increase in the frequency of $\mathrm{CD} 25^{+} \mathrm{Foxp} 3^{\mathrm{hCD} 2+}$ Treg cells in IAV-infected mice $10 \mathrm{dpi}$ when compared to PBS-treated controls (Fig. 2B). Conversely, the frequency of CD25-Foxp3 ${ }^{\text {hCD2- }}$ Tconv decreased significantly in thymi from IAV-infected mice compared to those from control mice (Fig. 2C). Surprisingly, unlike the numbers of Tconv, which were significantly reduced in atrophied thymi (Fig. 2C), the numbers of Treg cells were only slightly decreased, not reaching statistical significance (Fig. 2B). In addition, in thymi from IAV-infected mice at $10 \mathrm{dpi}$ the frequency of $\mathrm{CD} 25$ Foxp $3^{\mathrm{hCD} 2+}$ Treg cell precursors increased significantly compared to those from PBS-treated control mice, suggesting favored differentiation of Treg cells via these precursors in this setting (Fig. 2D).

When we tested the Treg cell and Tconv populations among recirculating Rag1 ${ }^{\text {GFP- CD4SP }}$ thymocytes, we neither observed an increase in frequencies nor numbers when thymi from IAV-infected mice $10 \mathrm{dpi}$ were compared to those from PBS-treated controls (Supporting Information Fig. $2 \mathrm{~B}+\mathrm{C}$ ), indicating that the increase in Treg cell frequency seen in thymi of IAV-infected mice does not result from an increased recirculation of Treg cells during infection-induced thymus atrophy. 
A

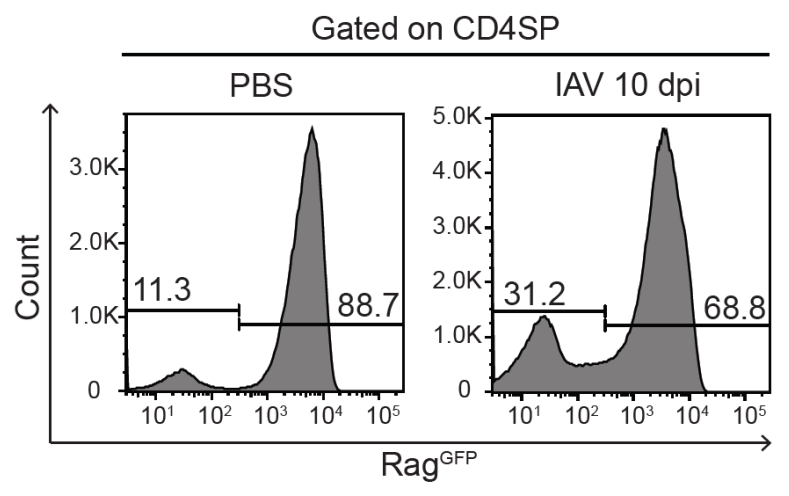

B Gated on CD4SP Rag ${ }^{\mathrm{GFP}+}$
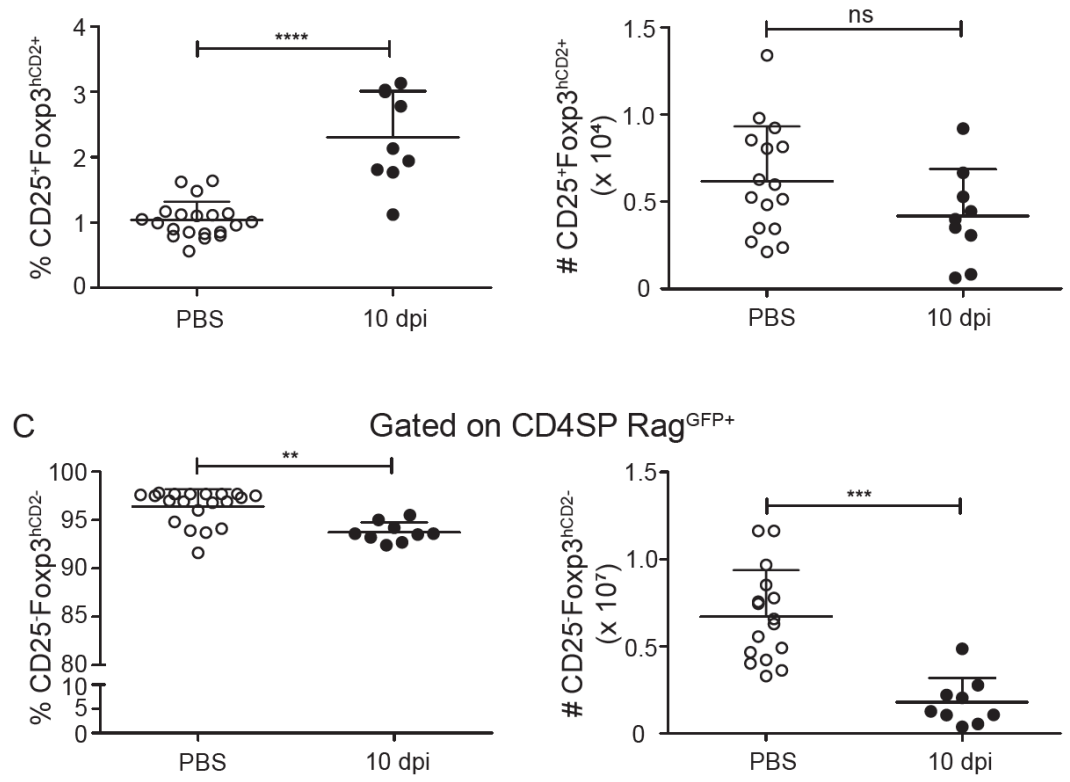

D
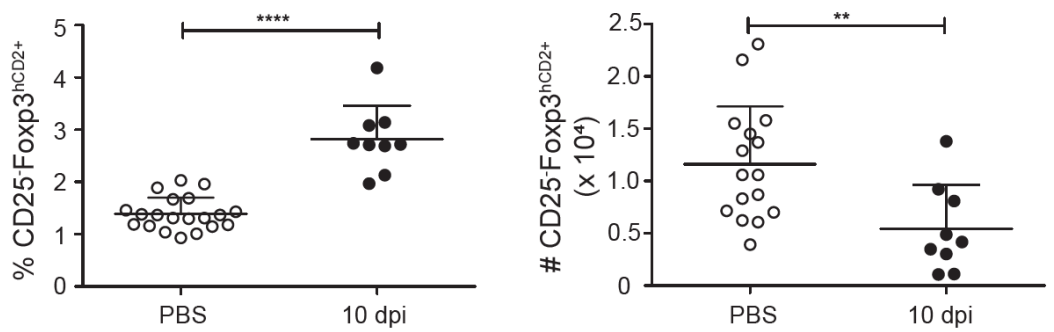

Figure 2. Frequency of de novo novo generated tTreg cells increases upon IAV infection. Foxp3 ${ }^{\text {hCD2}} x$ Rag $1^{\text {GFP }}$ double reporter mice were infected with IAV and control mice received PBS. Thymi were analyzed at different dpi. (A) Representative histograms depict Rag1 ${ }^{\text {GFP }}$ expression among CD4SP thymocytes from PBS-treated and IAV-infected mice at $10 \mathrm{dpi}$. Numbers indicate frequencies of cells in indicated gates. (B-D) Scatterplots summarize frequencies (left) and absolute numbers (right) of CD25+Foxp3 ${ }^{\text {hCD2+ }}$ Treg cells (B), CD25-Foxp3 ${ }^{\text {hCD2- }}$ Tconv (C) and CD25-Foxp3 ${ }^{\text {hCD2+ }}$ Treg cell precursors (D) among CD4SP Rag1 ${ }^{\text {GFP+ }}$ cells from PBS-treated (open circles) and IAV-infected (filled circles) mice at $10 \mathrm{dpi}$. Data were pooled from three independent experiments with 9-20 mice per group and presented as mean + SD. Each dot represents an individual mouse. Mann-Whitney test was used to test for statistical significance. 


\section{Intrathymic Treg cells and Tconv show comparable survival rates during IAV-induced thymus atrophy}

The data from the kinetic analysis indicate that newly generated tTreg cells are favored over their Tconv counterparts upon IAV-induced thymus atrophy. To get insights into the underlying molecular mechanism we first tested whether the relative increase of tTreg cells is a result of a superior ability to survive when compared to Tconv in the context of IAVinduced thymus atrophy by staining for active caspase 3/7. Examining the newly generated Rag1 ${ }^{\text {GFP+ }}$ CD4SP thymocytes revealed minimal propensity to apoptosis among both tTreg cells and Tconv as hardly any caspase $3 / 7^{+}$apoptotic cells could be detected (Fig. 3A). Interestingly, no impact of infection-induced thymus atrophy on survival of newly generated Treg cell and Tconv was observed at 7, 10 and $14 \mathrm{dpi}$, and both cell types showed similarly low frequencies of caspase $3 / 7^{+}$cells at all time points (Fig. 3B). In contrast, many more

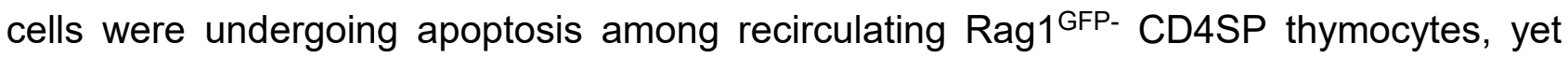
again no impact of infection-induced thymus atrophy and no differences between Treg cell and Tconv were observed (Fig. 3C+D). Together, these data indicate that the relative enrichment of newly generated tTreg cells is unlikely to result from a better survival compared to newly generated Tconv.

\section{Newly generated CD4SP thymocytes show unaltered TCR signaling upon IAV- induced thymus atrophy}

TCR signaling is critical for tTreg cell development $[3,31]$, and reduced TCR signaling has been shown to enhance Treg cell differentiation in multiple models [32-34]. To test whether IAV-induced thymus atrophy is accompanied by reduced TCR signaling, we sorted newly generated Rag $1^{\mathrm{GFP}+}$ CD4SP thymocytes from IAV-infected mice at $9 \mathrm{dpi}$, shortly before the peak of thymus atrophy, and stained them for CD5 and Nur77, the levels of which correlate with the intensity of TCR signaling and negative selection [35, 36]. Flow cytometric analysis revealed that Rag $1^{\text {GFP+ }}$ CD4SP thymocytes from IAV-infected mice showed similar mean fluorescence intensity levels for both CD5 and Nur77 when compared to newly generated Rag $1^{\text {GFP+ }}$ CD4SP thymocytes taken from PBS-treated control mice (Fig. 4A+B). Hence, these data suggest that a reduced TCR signaling is unlikely to be causative for the relative tTreg cell enrichment upon IAV-induced thymus atrophy. 


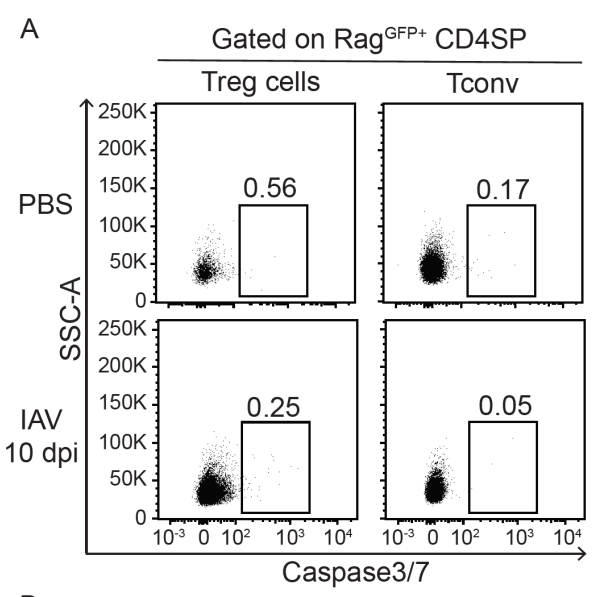

B

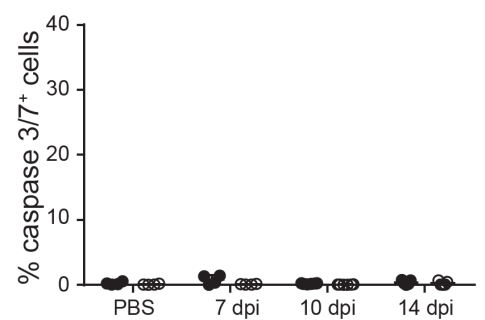

- Tconv

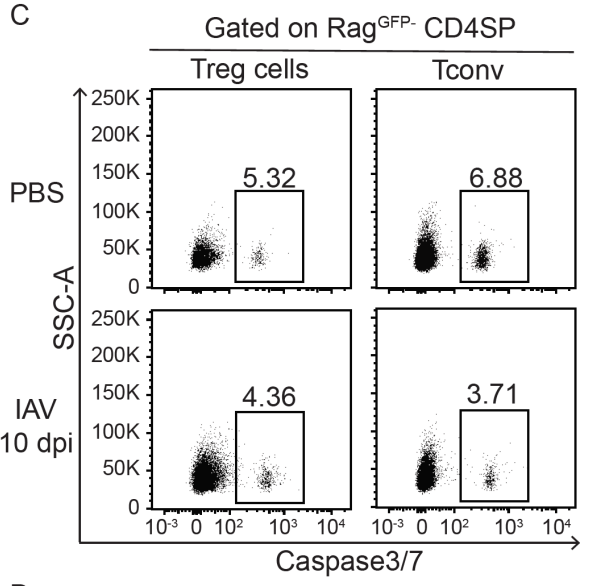

D

- Treg cells

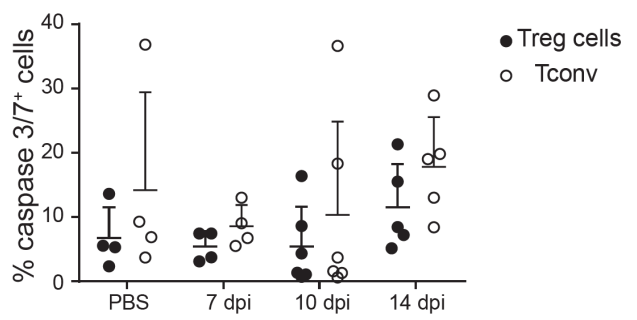

Figure 3. Intrathymic Tregs and Tconv show comparable survival rates during IAV-induced thymic atrophy. Foxp3 ${ }^{\text {hCD2}}$ Rag1 ${ }^{\text {GFP }}$ double reporter mice were infected with IAV and control mice received PBS. Thymi were analyzed at different dpi. (A) Representative dotplots depict caspase $3 / 7^{+}$cells among Treg cells and Tconv pregated on CD4SP Rag1 ${ }^{\text {GFP+ }}$ cells from thymi of PBS-treated and IAV-infected mice at $10 \mathrm{dpi}$. Numbers indicate fractions of cells in indicated gates. (B) Scatterplots summarize caspase $3 / 7^{+}$cells among Treg cells (filled circles) and Tconv (open circles) pregated on CD4SP Rag $1^{\text {GFP+ }}$ cells. (C) Representative dotplots depict caspase $3 / 7^{+}$cells among Treg cells and Tconv gated on CD4SP Rag1 ${ }^{\text {GFP- }}$ cells from thymi of PBS-treated and IAV-infected mice at $10 \mathrm{dpi}$. Numbers indicate fractions of cells in indicated gates. (D) Scatterplots summarize caspase $3 / 7^{+}$cells among Treg cells (filled circles) and Tconv (open circles) pregated on CD4SP Rag1GFP- cells. Data were pooled from two independent experiments with 4-8 mice per group and presented as mean + SD. Each dot represents an individual mouse. Mann-Whitney test was used to test for statistical significance.

A

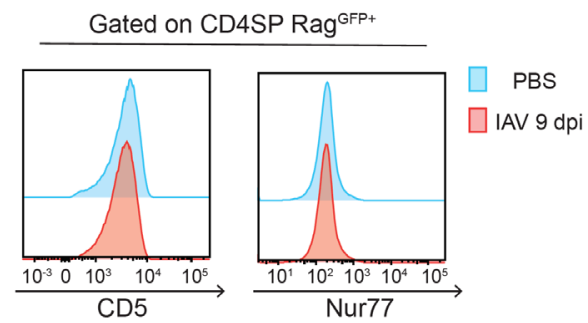

B

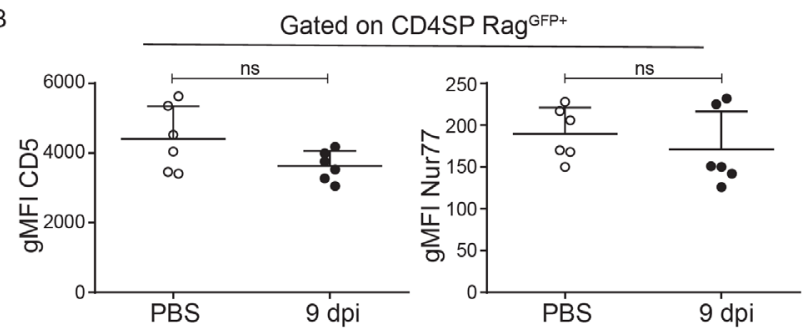

Figure 4. De novo generated CD4SP thymocytes show comparable levels of CD5 and Nur77 upon IAVinduced thymus atrophy. Foxp $3^{\text {hCD2}} \times$ Rag $1^{\text {GFP }}$ double reporter mice were infected with IAV and control mice received PBS. Thymi were collected, and CD4SP Rag $1^{\mathrm{GFP}+}$ cells were sorted and analyzed. (A) Representative histograms display CD5 (left) and Nur77 (right) expression in sorted CD4SP Rag1 ${ }^{\text {GFP+ }}$ cells from thymi of PBStreated (blue) and IAV-infected (red) mice at $9 \mathrm{dpi}$. (B) Scatterplots summarize geometric mean fluorescence intensity (gMFI) of CD5 (left) and Nur77 (right) in sorted CD4SP Rag $1^{\text {GFP+ }}$ cells from thymi of PBS-treated and IAV-infected mice at $9 \mathrm{dpi}$. Data were pooled from two independent experiments which included six mice per group and presented as mean + SD. Each dot represents an individual mouse. Mann-Whitney test was used to test for statistical significance. 


\section{Intrathymic levels of TGF- $\beta$ transiently increase upon IAV-induced thymus atrophy}

In addition to signals provided via the TCR also cytokines are essential mediators for Treg cell development in the thymus [37]. Thus, in order to assess the role of cytokines in the preferential increase of tTreg cells in the atrophied thymus, we measured their levels at different time points of IAV-induced thymus atrophy. Levels of IL-2, a key cytokine for tTreg cell development, were unchanged at 7,9 and 12 dpi when compared to PBS-treated controls (Fig. 5A). Similarly, levels for the inflammatory cytokines IL-1 $\alpha$, IL- $\beta$ and IFNy, all known to modulate tTreg cell development or be involved in thymus atrophy [22, 28], did not increase in the thymus upon infection-induced atrophy. Only slightly reduced IL-6 levels were observed in thymi at $12 \mathrm{dpi}$ when compared to PBS-treated controls (Supporting Information Fig. 3). Interestingly, levels of TGF- $\beta$, for which a positive effect on tTreg cell development was reported [38], were significantly elevated during infection-induced thymus atrophy at $7 \mathrm{dpi}$, before returning to levels of PBS-treated controls at $12 \mathrm{dpi}$ (Fig. 5B). These data suggest that increased levels of TGF- $\beta$ might contribute to an enhanced tTreg cell differentiation during IAV-induced thymus atrophy.

A

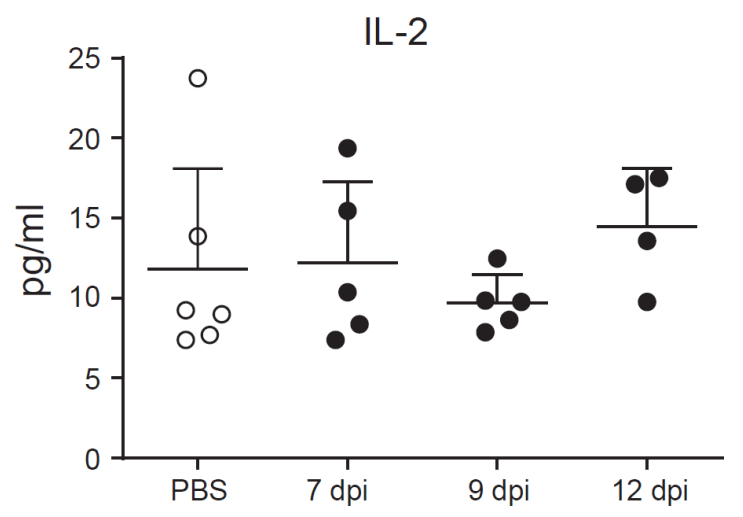

B

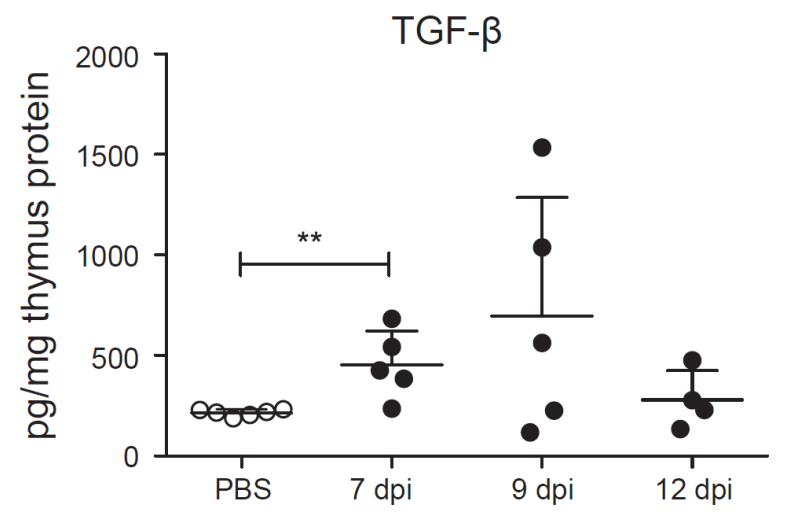

Figure 5. TGF- $\beta$ but not IL-2 in thymus increases significantly upon thymus atrophy. Foxp $3^{\text {hCD2 }} \times$ Rag $1{ }^{\text {GFP }}$ double reporter mice were infected with IAV and control mice received PBS. Thymi were analyzed at indicated dpi. Concentrations of IL-2 and TGF- $\beta$ were measured by Bio-plex 23-plex mouse kit or LEGENDplex kit, respectively. Scatterplots summarize IL-2 (A) and TGF- $\beta$ (B) concentrations in thymi from PBS-treated (open circles) and IAV-infected mice (filled circles) at indicated dpi. Data were pooled from two independent experiments with 4-6 mice per group and presented as mean + SD. Each dot represents an individual mouse. Mann-Whitney test was used to test for statistical significance. 


\section{Mathematical modeling suggests increased DP thymocyte death and augmented exit of SP thymocytes as cause for the increased frequency of tTreg cells upon IAV- induced thymus atrophy}

Next, we analyzed possible mechanisms underlying the relative enrichment of newly generated tTregs upon IAV-induced thymus atrophy with a mathematical model simulating the time evolution of thymocyte populations during IAV infection, including Treg cells and their precursors, using the data from the kinetic study (Fig. 6A). Each node represents a population and arrows represent the mechanisms of differentiation, proliferation or death (for details see 'Design of the mathematical model' in the Material and Methods section). The DP thymocyte compartment is built on a previous model of T cell development and population dynamics, where the early proliferating DP (eDP) and late, resting DP thymocytes (IDP) are distinguished [17]. We added the following Rag1+ CD4SP thymocyte populations: CD25-Foxp3- cells (Tconv), CD25 ${ }^{+}$Foxp3- Treg cell precursors, CD25-Foxp3 ${ }^{+}$Treg cell $^{-}$ precursors, and $\mathrm{CD}^{2} 5^{+}$Foxp $3^{+}$mature Treg cells. Furthermore, as several studies have highlighted that the two Treg cell precursor populations arise at different time points of $\mathrm{T}$ cell development and harbor differential dynamics [4-6, 27], we compared different model structures for Treg cell development (Supporting Information Fig. 4), namely where both Treg cell precursors either arise from DP thymocytes (structure A) or Tconv (structure B), or where CD25-Foxp3 ${ }^{+}$Treg cell precursors arise from Tconv, while CD25 ${ }^{+}$Foxp3- Treg cell precursors directly arise from DP thymocytes (structure C).

The effect of IAV infection is modeled as a perturbating factor $F$, with a Gaussian dynamic over time (Fig. 6B), whose peak time and width are to be found. To discriminate possible mechanisms responsible for the dynamics of the thymocyte populations during IAV-induced thymus atrophy, we tested different assumptions, namely Factor F increases DP thymocyte death, Factor $\mathrm{F}$ increases de novo CD25-Foxp3 ${ }^{+}$Treg cell precursor generation, Factor $\mathrm{F}$ increases the export of all SP thymocyte populations equally, or Factor $F$ increases the export of Tconv compared to Treg cells and Treg cell precursors differentially (Fig. 6B). Deliberately, we did not test an increased cell death among Tconv or Treg cell precursors, since we did not detect any significant cell death among newly generated CD4SP thymocyte subsets (Fig. 3). Fitting the data under these assumptions identified the increase in DP thymocyte death as a dominant factor that could already explain for the most data the dynamics of all major thymocyte populations, including the transient increase in the 
frequency of mature tTreg cells and their CD25-Foxp3 ${ }^{+}$precursors during the peak of the IAV-induced thymus atrophy (green curve in Fig. 6C-H and Supporting Information Fig. 5-7). If other assumptions like an increased de novo CD25-Foxp3 ${ }^{+}$Treg cell precursor generation or an increased export of all SP thymocyte populations are simulated alone, the DP thymocyte shrinkage dynamics could not be explained, as can be seen by the very high costs (Supporting Information Fig. 8).

While increased DP thymocyte death was compatible with most data for the three model structures, the peaks of the fractions of mature tTreg cells and their CD25-Foxp $3^{+}$precursors appeared earlier and were slightly higher in the experimental measurement when compared to the simulations (green curve in Fig. 6C-H and Supporting Information Fig. 5-7). Thus, we tested combinations of assumptions.

At first, we simulated atrophy under both increased DP thymocyte death and increased de novo generation of CD25-Foxp3 ${ }^{+}$Treg cell precursors (hypothesis 2 in Fig. 6C-H, yellow curves). However, the best parameter values (see Supporting Information Fig. 9 and 'Design of the mathematical model' in the Material and Methods section) suggests an only marginally 1.00006-fold increased de novo generation of CD25-Foxp3 ${ }^{+}$Treg cell precursors (yellow curve in Fig. 6 partially overlapping with the green curve, and Supporting Information Fig. 5+6). A sensitivity analysis around the best curve showed that a substantially modulated de novo generation of CD25-Foxp3 ${ }^{+}$Treg cell precursors would be detectable due to different dynamics of the Treg cell precursors (Supporting Information Fig. 10A). The corresponding identifiability analysis showed that the increased de novo generation of CD25-Foxp ${ }^{+}$Treg cell precursors also increases the cost of the fittings as it has a strong impact on the dynamics of absolute cell numbers (Supporting Information Fig. 10B). Thus, increased de novo generation of CD25-Foxp $3^{+}$Treg cell precursors does not provide a better fit nor explanation of the dataset (Supporting Information Fig. 9).

We next tested an increased export of all SP thymocytes (CD8SP thymocytes, Tconv, Treg cells and both Treg cell precursors) with equal strength and simulated this together with the assumption of an increased DP thymocyte death (hypothesis 3 in Fig. 6B). This is supported by the observation that the number of CD8SP thymocytes and Tconv seems to decay before the predicted effect of the increased DP thymocyte death (green curve in Fig. 6D+E). The best fit improved the dynamics of CD8SP thymocytes and Tconv, and also the transient increase in the frequency of mature tTreg cells and their CD25-Foxp ${ }^{+}$precursors was 
refined (blue curves in Fig. 6D+E+G+H and Supporting Information Fig. 5+6). In general, the cost of each model structure was improved when compared to the assumption of an increased DP thymocyte death alone, and the best fit with model structure B had a significantly better corrected Akaike Information Criterion (AICc) when compared to the other model structures (Supporting Information Fig. 9).

Finally, we assumed a differential increase of the export of Tconv in comparison to Treg cells and their precursors, and simulated this together with increased DP thymocyte death (hypothesis 4). Although the cost of the best parameter set for this highly intuitive hypothesis was lower, indicating a better quantitative fitting (Supporting Information Fig. 9), the dynamics of the mature tTreg cells and their CD25-Foxp $3^{+}$precursors around the peak of the IAV-induced thymus atrophy were less well explained (red curves in Fig. $6 \mathrm{G}+\mathrm{H}$ and Supporting Information Fig. 5+6). This discrepancy comes from the latest time point, where hypothesis 4 fits best, particularly regarding the absolute cell numbers, at the expense of a less good fit around the peak of the IAV-induced thymus atrophy.

For each model structure, hypotheses 3 and 4 are most well suited to describe the dynamics of all thymocyte populations during IAV-induced thymus atrophy (Supporting Information Fig. 9). While in model structure $A$ and $C$, a differential export of Tconv and Tregs (hypothesis 4) does not impove the AICc index, model structure B shows an improvement of the AICc in this case. In order to discriminate between hypotheses 3 and 4, we predicted the half-life of both Treg cell precursor populations from the obtained best parameter sets, as an independent qualitative validation. In line with published data [5], hypothesis 3 within model structure B predicted realistic values of 0.5, 3.1 and 3.8 days half-life for CD25+Foxp3precursors, CD25-Foxp3+ precursors and mature Treg cells, respectively, while hypothesis 4 generated values out of range (Supporting Information Fig. 9). We therefore rejected hypothesis 4 and kept model structure B with hypothesis 3 as the most likely scenario. In conclusion, the assumption of an increased DP thymocyte death is the major determinant for the dynamics of the thymocyte populations during IAV-induced atrophy, together with the assumption of an increased export of all SP thymocyte populations with equal strength. Furthermore, model structure B, where both Treg cell precursors arise from Tconv, is the best model structure in terms of cost, AICc values, and realistic half-lives of thymocyte subsets. 
A

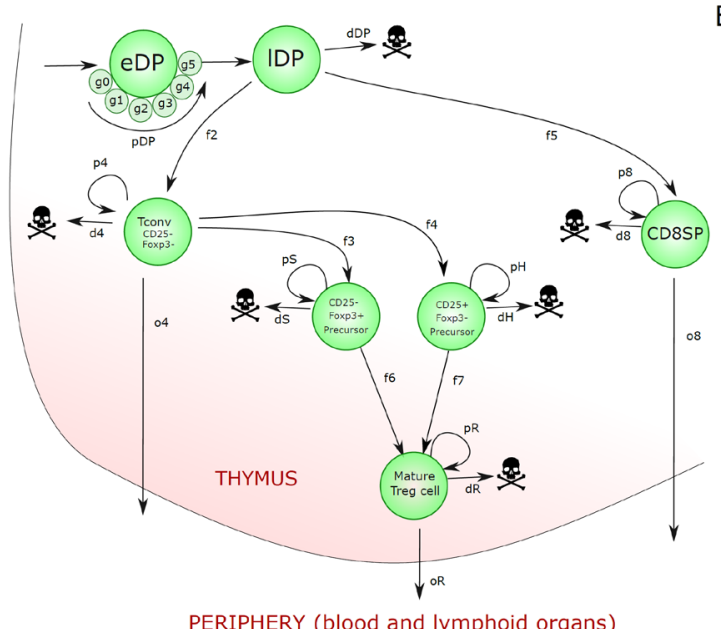

PERIPHERY (blood and lymphoid organs)
$\mathrm{B}$

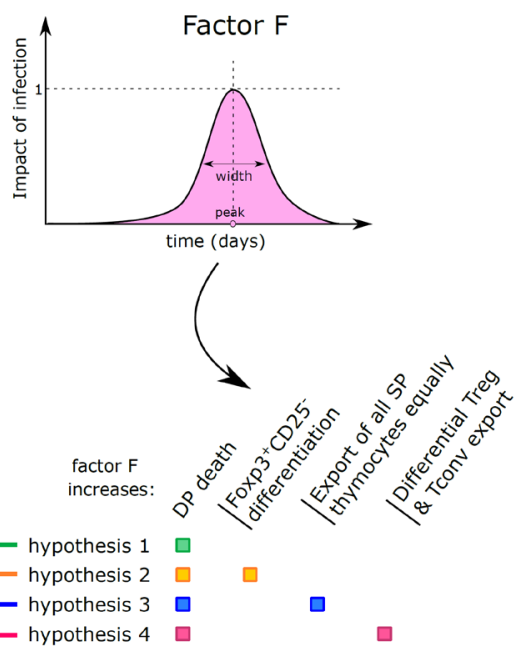

C
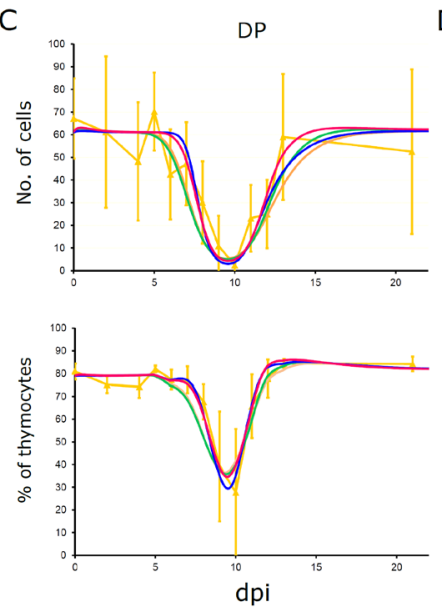

$\mathrm{F}$
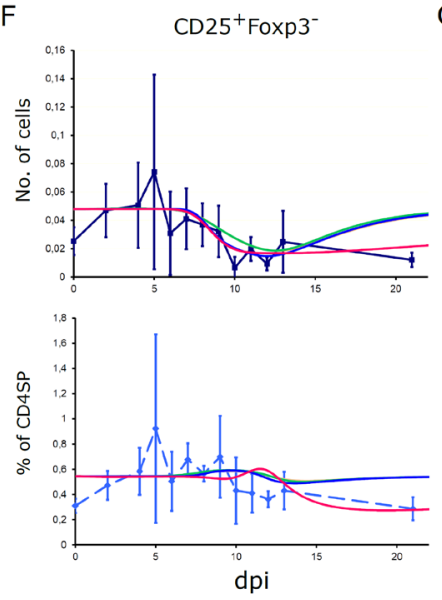

D
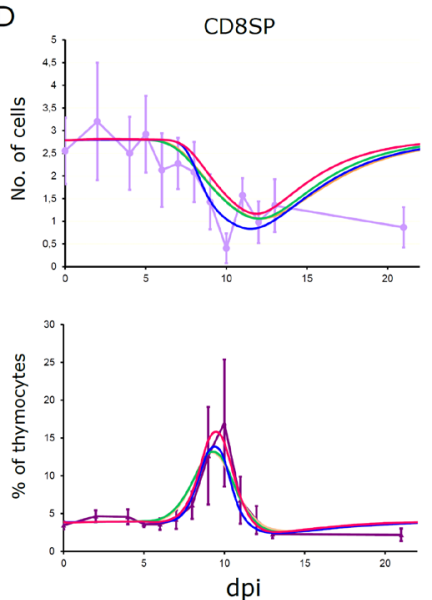

G
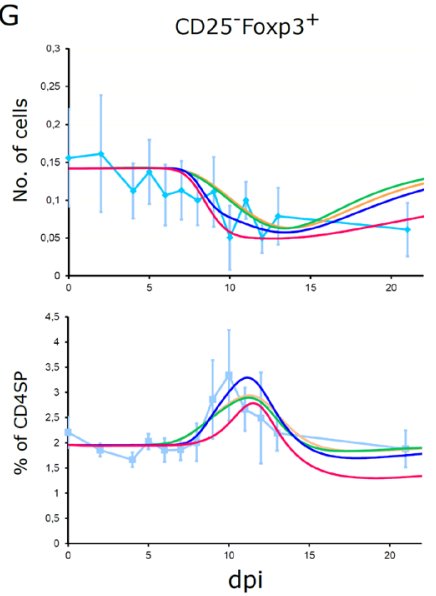

$E$
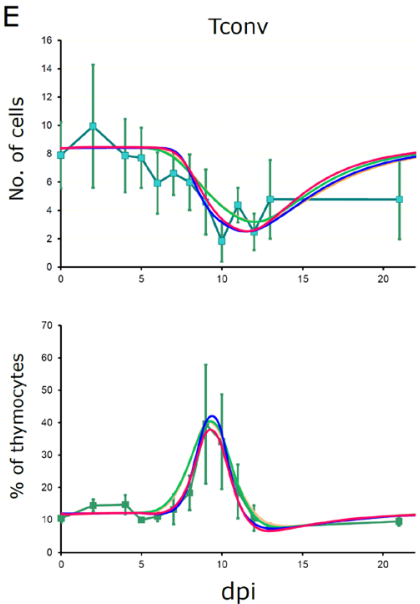

$\mathrm{H}$
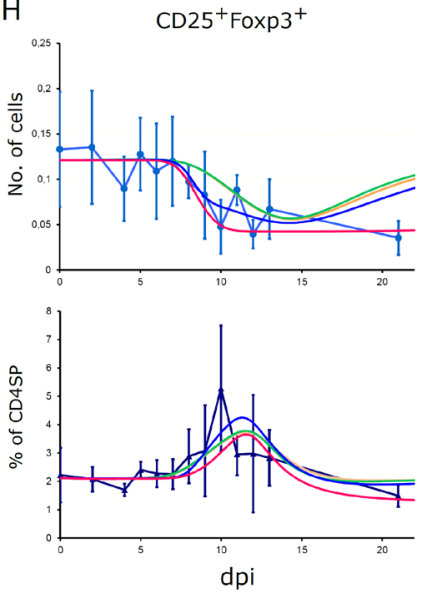

Figure 6. Dynamical modeling of thymus kinetics under IAV-induced atrophy under different hypotheses. (A) Mathematical model for DP thymocyte, Tconv, CD8SP thymocyte, Treg cell precursors and mature Treg cell compartments. (B) (Top) Factor F, used to model the effect of IAV infection on the thymus. (Bottom) Different hypotheses on the effect of IAV infection. (C-H) Best fitted curves to explain experimental kinetics of all subpopulations with each hypothesis, as absolute numbers (top) and frequencies (bottom). The colors of the curves match the hypotheses in B. Absolute numbers are only shown for the newly generated Rag $1^{\mathrm{GFP}+}$ populations. Frequencies show the summed percentage of Rag $1^{\mathrm{GFP}+}$ and Rag $1^{\mathrm{GFP}-}$ cells together. 


\section{IAV-induced thymus atrophy results in reduced TCR repertoire diversity among newly produced thymocytes}

We next asked whether the IAV-induced thymus atrophy also affects the TCR repertoires of newly produced Treg cells and Tconv. To this end, CD25 ${ }^{+}$Foxp $3^{\text {hCD2+ }}$ Treg cells and CD25Foxp3 ${ }^{\text {hCD2- }}$ Tconv were isolated from both newly produced Rag $1^{\text {GFP+ }}$ as well as recirculating Rag1 ${ }^{\text {GFP- }}$ CD4SP thymocytes of PBS-treated or IAV-infected mice 10 dpi. RNA was isolated from sorted cells and subjected to high-throughput next-generation sequencing of Tcrb transcripts to assess the TCR repertoire. Remarkably, the TCR repertoire diversity of the newly produced Treg cells was significantly reduced upon IAV infection (Fig. 7A) and was accompanied by a reduction of the rare clones (Fig. 7B). A similar pattern emerged when observing the newly produced Tconv, although not reaching statistical significance (Fig. 7A+B). Yet, the impact of IAV infection on the TCR repertoire diversity was less pronounced among the recirculating Treg cells and Tconv (Fig. 7A+B). Together, these data suggest that IAV-induced thymus atrophy causes a reduction in the TCR repertoire diversity of newly produced CD4SP thymocyte subsets, while having much less influence on the TCR repertoire of the recirculating $T$ cell subsets.
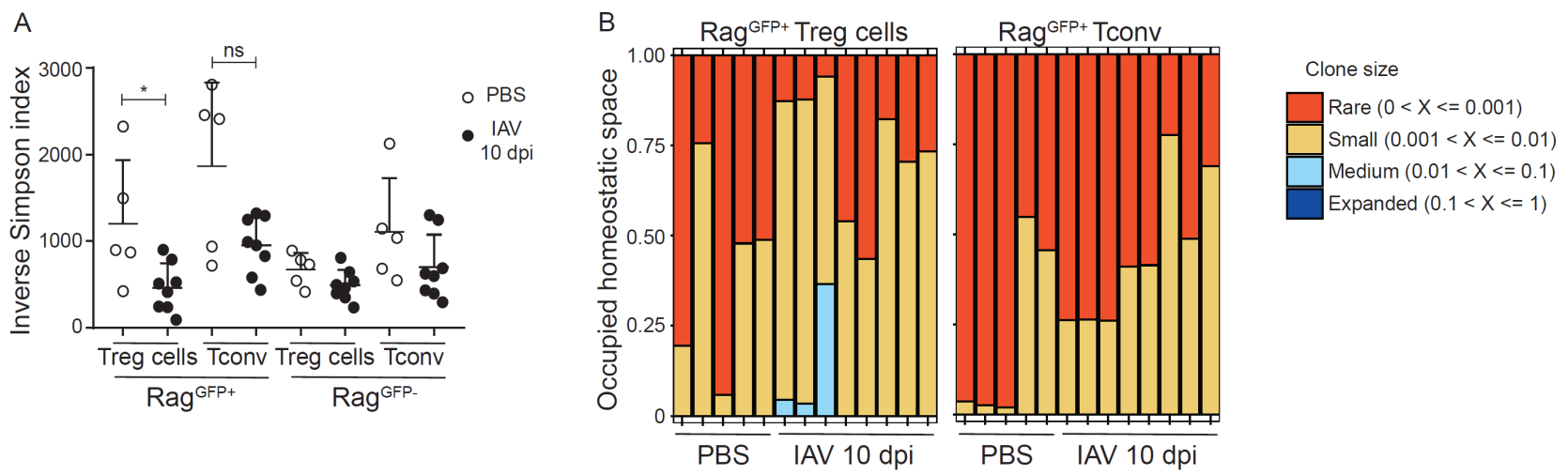

Figure 7. IAV-induced thymus atrophy results in reduced TCR repertoire diversity among de novo generated T cells. Foxp $3^{\mathrm{hCD}} \mathrm{x}$ Rag $1^{\mathrm{GFP}}$ double reporter mice were infected with IAV and control mice received PBS. Thymi were collected at $10 \mathrm{dpi}$, Rag $1^{\mathrm{GFP}+} \mathrm{CD} 25^{+} \mathrm{Foxp} 3^{\mathrm{hCD} 2+}$, Rag $1^{\mathrm{GFP}+} \mathrm{CD} 25$ Foxp ${ }^{\mathrm{hCD} 2-}$, Rag $1^{\mathrm{GFP}-}$

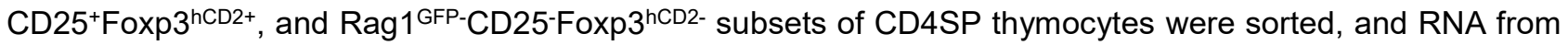
these cells was subjected to next-generation sequencing (NGS) of Tcrb transcripts. (A) Scatterplot summarizes the inverse Simpson index of thymic Rag1 $1^{\text {GFP+ }}$ Treg cells, Rag1 ${ }^{\text {GFP+ }}$ Tconv, Rag1 ${ }^{\text {GFP- Treg cells }}$ and Rag1GFP- Tconv isolated from either PBS-treated (open circles) or IAV-infected (filled circles) mice at $10 \mathrm{dpi}$. Each dot represents an individual mouse. (B) Cumulative bar graphs depict clonal homeostasis of Rag1 ${ }^{\text {GFP+ }}$ Treg cells and Rag1 ${ }^{\text {GFP+ }}$ Tconv sorted from thymi of PBS-treated and IAV-infected mice at $10 \mathrm{dpi}$. Each bar represents an individual mouse. Data were pooled from two independent experiments with 5-8 mice per group and presented as mean + SD. Mann-Whitney test was used to test for statistical significance. 


\section{DISCUSSION}

In the present study, we have shown that the massive, yet transient IAV-induced thymus atrophy is accompanied by transiently increased frequencies of mature tTreg cells and their CD25-Foxp $3^{+}$precursors. This increase mapped to the newly generated Rag $1^{\text {GFP+ }}$ Treg cells, and interestingly, while Rag1 ${ }^{\text {GFP+ }}$ Tconv were significantly reduced in terms of both proportion and numbers, numbers of Rag1GFP+ Treg cells were largely unaffected. Nonetheless, the relative increase of Treg cells was not the result of a better survival, as both Rag1 $1^{\text {GFP+ }}$ Treg cells and Tconv demonstrated similar (and negligible) propensity to apoptosis following IAV-induced thymus atrophy. Yet, the TCR repertoire diversity of both newly produced tTreg cells and Tconv was reduced upon IAV-induced thymus atrophy. Mathematical modeling suggested that the infection-induced increased death of DP thymocytes is the major determinant for the dynamics of the thymocyte populations during IAV-induced atrophy. It further proposed that an augmented exit of SP thymocytes could be involved in better describing the dynamics, rather than an enhanced tTreg cell generation being responsible for the relative increase in tTreg cells upon IAV-induced thymus atrophy. Our data argue for a model in which IAV-induced thymus atrophy differentially affects the dynamics of tTreg cells and Tconv.

Several previous reports had described the propensity of IAV infection to induce thymus atrophy [21-23]. Although different mechanisms have been suggested to contribute to thymus atrophy induced by IAV infection, such as IFNY produced by NK cells or innate $\mathrm{CD} 8 \alpha^{+} \mathrm{T}$ cells, they all cause a loss of DP thymocytes, which is thought to result from an increase in serum corticosteroids [8]. Consistent with our data, a rapid rebound occurs and the thymus regains its usual shape and cellularity. Interestingly, IFNy levels in thymi were not elevated upon infection in our experimental model, suggesting that IFNy might not be essential in IAV-induced thymus atrophy. Since Liu et al. and Duan et al. injected IFNYneutralizing antibodies intraperitoneally $[22,23]$, it is possible that this neutralization attenuated the degree of inflammation, which subsequently reduced the severity of thymus atrophy. It remains to be defined whether intrathymic injection of IFNy would have a similar alleviating effect on thymus atrophy.

Although thymic cellularity in the context of thymus atrophy was significantly decreased, the number of tTreg cells did not significantly change. In contrast, for Tconv both the frequency and absolute number were significantly reduced. This is thought to be deleterious to the 
host, since it is usually accompanied by lymphopenia [21]. In the context of IAV-induced thymus atrophy, the thymus may actively spare Treg cells in order to dampen the immune response after the peak of infection has passed. However, it is also possible that Treg cells are kept under tight control because their decrease, even in a transient manner, might result in the occurance of autoimmune episodes.

The finding that tTreg cells increase relative to Tconv upon IAV-induced thymus atrophy may be explained by various mechanisms, including a better survival of Treg cells over their Tconv counterparts. Yet, we could experimentally exclude this possibility by staining thymocytes for caspase 3/7. Interestingly, we saw a negligible fraction of cells undergoing apoptosis upon gating on Rag $1^{\mathrm{GFP}+}$ cells, which represent the newly generated thymocytes, and furthermore, the frequency of caspase $3 / 7^{+}$Treg cells was similar to that of Tconv. Intriguingly, apoptosis was most evident among the recirculating (Rag1 ${ }^{\text {GFP-) }}$ T cell subsets, and it will be very interesting to unravel the molecular mechanism underlying this differential susceptibility towards apoptosis between newly generated thymocytes and recirculating $\mathrm{T}$ cells in future studies.

The finding that tTreg cell generation is enhanced in the context of thymus atrophy has been shown before in a model of accelerated thymus atrophy [33]. In this model, accelerated thymus atrophy resulting from the induced deletion of FoxN1 was accompanied by disruption of negative selection [39]. The authors argue that the atrophied thymus attempts to balance this defect by actively generating tTreg cells. Indeed, they propose the decreased TCR signaling described to occur in this model to be the reason for this enhanced generation of tTreg cells [33]. Yet, in our model, the intensity of CD5 and Nur77 expression remained unchanged upon thymus atrophy. Furthermore, we had observed a significant increase in the frequency of only CD25-Foxp3 ${ }^{+}$, but not CD25+Foxp3- Treg cell precursors, and Farrar and colleagues have recently demonstrated that CD25-Foxp $3^{+}$precursors rely on a high TCR signal strength for their differentiation [6]. Thus, it is highly unlikely that a reduced TCR signaling is causative for the relative tTreg cell enrichment upon IAV-induced thymus atrophy.

The role of TGF- $\beta$ in tTreg cell development is still a matter of debate. Initially, TGF- $\beta$ signaling in the thymus was thought to be indispensable [38]. However, other reports claimed otherwise [40] or that it was only required for the survival of tTreg cells [41]. In 2014, Konkel et al. showed that apoptosis in the thymus is driving tTreg cell generation through 
TGF- $\beta$ [42]. Since infection-induced thymus atrophy is accompanied by apoptosis of thymocytes, especially DP thymocytes [8], we asked whether TGF- $\beta$ levels are increased in the context of IAV-induced thymus atrophy. Indeed, TGF- $\beta$ levels increased significantly upon thymus atrophy. However, so far experimental data demonstrating a contribution of these increased TGF- $\beta$ levels to an enhanced tTreg cell generation during IAV-induced thymus atrophy are missing. Furthermore, mathematical modeling did not provide any evidence for an involvement of this mechanism in the relative increase in tTreg cells upon IAV-induced thymus atrophy. Instead, the model suggested that the infection-induced increased death of DP thymocytes is the main driving factor, plus a possible contribution of an augmented exit of all SP thymocyte populations.

To the best of our knowledge, so far no mathematical model has accounted for the dynamic development of Treg cell precursors in the thymus. Despite the fact that a lot of progress has been made regarding our understating of tTreg cell development during the past years [2-6, 24-29], it is still not completely understood at which stage of T cell development the two Treg cell precursor populations arise, and it has been suggested that even earlier preprecursors do exist [43]. Here, we show that the dynamics upon thymus atrophy contain information on Treg cell precursor dynamics. Further work would need to include quantitative datasets on the Treg cell populations' properties, such as half-lives, BrdU labeling, or intrathymic injection of Treg cell precursors, to experimentally validate the model. Further, although all model structures concord to delineate DP thymocyte death as the major determinant for the dynamics of the thymocyte populations during IAV-induced atrophy, the mechanistic impact of SP thymocyte export was leading to a better fit, judged by cost and AICc values. Since model structure B is the structure with the best fitting cost, our mathematical model is providing a first hint that Treg cell precursors might arise from CD4SP rather than directly from DP thymocytes.

The finding that CD25-Foxp3 ${ }^{+}$and CD25 $^{+}$Foxp3- Treg cell precursors have different dynamics of Foxp3 induction [4-6, 27] might indicate that they recognize different types of self-antigens. We have previously shown that thymic selection can be simulated at singlecell level on the basis of time-integrated signaling to discriminate between Tconv and Treg cell differentiation [44]. It would be interesting to investigate if this thymic selection model would predict the diversity of tTreg cells and their precursors during infection-induced thymus atrophy. 
The data of the present study demonstrate that IAV-induced thymus atrophy results in a decreased diversity of the TCR repertoire only among the newly generated T cells, both Treg cells and Tconv. Recirculating T cells were only slightly affected in terms of their TCR repertoire diversity. These data further support the notion that IAV-induced thymus atrophy is primarily affecting the process of $\mathrm{T}$ cell development and has only limited impact on recirculating $\mathrm{T}$ cells during and shortly after the infection. The narrowing of the TCR repertoire diversity could be a direct consequence of DP thymocyte death or the result of a functional impairment of thymic antigen-presenting cells (APCs) or simply a decrease in their numbers secondary to IAV-induced thymic atrophy. Thymic APCs are well known to critically contribute to tTreg cell development [45-49], and future studies need to unravel if IAVinduced thymus atrophy results in alterations of the thymic dendritic cell and thymic epithelial cell compartment, which finally affect the TCR repertoire diversity of newly generated T cell subsets. Indeed, it was shown in models of accelerated thymus atrophy and thymus atrophy due to aging that the functionality of thymic APCs was negatively influenced, resulting in a reduced ability to present self-antigens, which perturbed negative selection and finally provoked autoimmune phenotypes [50].

In summary, we have shown that IAV-induced thymus atrophy is strongly affecting the dynamics of major thymocyte populations, finally resulting in a relative increase of tTreg cells with an altered TCR repertoire. Our findings are particularly relevant given the frequent occurrence of thymus atrophy in different settings. It remains to be defined whether the transiently altered Treg cell/Tconv ratio has an effect on the peripheral homeostasis of these cells, especially when the cause of this atrophy is more protracted or more frequently taking place. Since thymus atrophy has been reported to occur in the context of chronic infections [8] and cancer [51,52], it would be interesting to investigate the long-lasting consequences of thymus atrophy on Treg cell homeostasis and function in those settings. 


\section{MATERIAL AND METHODS}

\section{Animals}

Foxp3 $3^{\text {hCD2 }} \times$ Rag1 ${ }^{\text {GFP }}$ reporter mice (C57BL/6 background) $[30,53]$ were bred and maintained at the animal facility of the Helmholtz Centre for Infection Research (Braunschweig, Germany). All mice were kept under specific pathogen-free conditions. In all experiments, female mice at the age of six weeks were used. All animal experiments were conducted in compliance with the German animal protection law (TierSchG BGBI. I S. $1105 ; 25.05 .1998)$. Animals were housed and handled in accordance with good animal practice as defined by FELASA and the national animal welfare body GV-SOLAS. All animal experiments were approved by the Lower Saxony Committee on the Ethics of Animal Experiments as well as the Lower Saxony State Office of Consumer Protection and Food Safety under the permit number 33.19-42502-04-15/2058. All experiments were performed in accordance with the institutional, state, and federal guidelines.

\section{Virus}

For the infection experiments, the mouse-adapted strain PR8M of IAV H1N1 was used. The virus was grown on MDCK cell line, harvested as a supernatant of the cultures, aliquoted and stored at $-80^{\circ} \mathrm{C}$. 666 ffu were given intranasally in $20 \mu \mathrm{PBS}$ to Foxp $3^{\text {hCD2}} \times$ Rag $1^{\text {GFP }}$ reporter mice. Control mice received an intranasal application of $20 \mu \mathrm{IPBS}$.

\section{Cell sorting}

CD4SP thymocyte subsets were sorted from thymi of control and infected mice. Thymic single-cell suspensions were enriched for CD4SP thymocytes by means of staining, using CD8a-APC antibody (53-6.7, purchased from Biolegend), followed by labeling with anti-APC microbeads (Miltenyi Biotec). DP and CD8SP thymocytes were depleted by applying the "depleteS" program of AutoMACS Pro (Miltenyi Biotec). Enriched CD4SP thymocytes were then stained with CD4-Pacific blue (RM4-5) fluorochrome-conjugated antibodies (BioLegend). FACS Aria Ilu and FACS Aria II SORP (BD Biosciences) were employed for cell sorting of CD4SP thymocyte subsets. 


\section{Antibodies and flow cytometry}

Flow cytometric analysis was performed as described recently [54]. Cell suspensions isolated from thymi were labeled directly with the following fluorochrome-conjugated antimouse antibodies purchased from either BioLegend, BD Biosciences, or eBioscience: CD4BV605 (RM4-5), CD8a-APC (53-6.7), CD25-PerCP-Cy5.5 (PC61.5), and human CD2BV421 (RPA-2.10). Detection of active caspase $3 / 7$ was accomplished using CellEvent ${ }^{\mathrm{TM}}$ Caspase-3/7 Red Detection Reagent (ThermoFisher Scientific). Sorted CD4SP Rag1 ${ }^{\text {GFP+ }}$ thymocytes were stained with CD5-PerCP-Cy5.5 (53-7.3) and Nur77-PE (12.14) antibody (both from eBiosciences). Exclusion of dead cells was done by using the LIVE/DEAD ${ }^{\text {TM }}$ Fixable Blue stain kit (Invitrogen). Cells were acquired on LSR Fortessa ${ }^{\text {TM }}$ flow cytometer (BD Biosciences), and data were analyzed with FlowJo software (BD Biosciences).

\section{Measurements of cytokines in the thymus}

Thymi isolated from Foxp3 $3^{\mathrm{hCD} 2} \times \mathrm{Rag}^{\mathrm{GFP}}$ reporter mice were homogenized and lysed using Bio-Plex Cell Lysis Kit (BioRad Laboratories), and the protein content of the thymic lysates was measured using Pierce ${ }^{\text {TM }}$ BCA Protein Assay Kit (Thermo Scientific). Afterwards, equal protein amounts of the lysates $(25 \mu \mathrm{g})$ were applied to the Bio-Plex Mouse Cytokine 23-plex Assay Kit (Bio-Rad Laboratories) and measured with the BioPlex200 system and BioPlexManager 6.2 according to manufacturer's instructions. For the measurement of TGF- $\beta$ levels in thymus, the same lysates were processed using LEGENDplex ${ }^{\mathrm{TM}}$ Mouse/Rat Free Active/Total TGF- $\beta 1$ Assay (BioLegend) according to manufacturer's instructions. Samples were then acquired using LSRFortessa ${ }^{\mathrm{TM}}$ flow cytometer, and data were analyzed with FlowJo software. TGF- $\beta$ concentrations were normalized to thymic protein concentrations.

\section{Design of the mathematical model}

The development of all major thymocyte populations is modeled as a set of Ordinary Differential Equations (ODEs). Three model structures were considered with different Treg cell differentiation pathways (see Supporting Information Fig.4). The equations and parameters from the DP thymocyte compartment were directly taken from Thomas-Vaslin et al. [17]. The early proliferating DP $(e D P)$ thymocytes were separated into generations $\left(G_{0} \ldots G_{N \max }\right)$, while late DP $(I D P)$ thymocytes are a unique population of cells dying or differentiating into SP thymocytes. The total DP thymocyte pool size was resized to 61.2 
million cells as measured in the present study. The equations were adapted using a continuous number of divisions and a fraction of cells performing the last division, such that the proliferation rate can be made dependent on thymus size. By defining a maximum $N_{\max }$ of generations to be simulated, the equations for the eDP thymocyte pool follow:

$$
\begin{aligned}
& \frac{d\left[G_{0}\right]}{d t}=I \rho_{-1}-\left(p_{\mathrm{eDP}}+d_{\mathrm{eDP}}\right)\left[G_{0}\right] \\
& \frac{d\left[G_{1}\right]}{d t}=2 p_{\mathrm{eDP}} \rho_{0}\left[G_{0}\right]-\left(p_{\mathrm{eDP}}+d_{\mathrm{eDP}}\right)\left[G_{1}\right] \\
& \ldots=2 p_{\mathrm{eDP}} \rho_{\mathrm{i}-1}\left[G_{\mathrm{i}-1}\right]-\left(p_{\mathrm{eDP}}+d_{\mathrm{eDP}}\right)\left[G_{\mathrm{i}}\right] \\
& \frac{d\left[G_{\mathrm{i}}\right]}{d t} \ldots=2 p_{\mathrm{eDP}} \rho_{\mathrm{N}_{\max }-1}\left[G_{\mathrm{N}_{\max }-1}\right]-\left(p_{\mathrm{eDP}}+d_{\mathrm{eDP}}\right)\left[G_{\mathrm{N}_{\max }}\right] \\
& \frac{d\left[G_{\mathrm{N}_{\max }}\right]}{d t}=\sum_{i=0}^{N_{\max }}\left[G_{\mathrm{i}}\right] \\
& {[e D P] }
\end{aligned}
$$

where

$$
\rho_{i \in\left[-1 . . N_{\max }\right]}=\left\{\begin{array}{l}
1 \text { if } i<N \\
n_{\mathrm{div}}-N \text { if } i=N-1 \\
0 \text { if } i \geq N
\end{array}\right.
$$

I denotes the inflow of cells from DN thymocytes. The population $G$ represents eDP thymocytes that are proliferating, but did not complete yet the $i+1^{\text {th }}$ cell cycle. With $N=$ floor $\left(n_{\text {div }}\right)<N_{\max }$, an average of $n_{\text {div }}$ divisions is modeled with a fraction $n_{\text {div }}-N$ of cells actually entering the $N^{\text {th }}$ cycle $\left(G_{N}\right)$ while the remaining fraction exits to the IDP thymocyte stage. It appears as the fraction $\rho_{i}$ of cells leaving $G_{i}$ that are allowed to enter a new cell cycle, which is 1 for each division until $N-2$, a fraction for the $G_{N-1}$, and 0 afterwards until GNmax. The proliferation rate peDP denotes the rate of cells that succeed to reach the next generation, which appear as twice as many in the next generation. The flow of cells leaving the eDP pool to enter the IDP population is therefore:

$$
F_{\mathrm{eDP} \rightarrow \mathrm{IDP}}=\sum_{i=0}^{N_{\max }} 2\left(1-\rho_{\mathrm{i}}\right) p_{\mathrm{eDP}}\left[G_{\mathrm{i}}\right]
$$

The differentiation between populations is taken as a linear process. The differentiation rates from IDP thymocytes into Tconv and CD8SP thymocytes are called $f_{2}$ and $f_{5}$, respectively. 
Depending on the model structure, Foxp3 ${ }^{+} \mathrm{CD} 25^{-}$and Foxp3-CD25 $5^{+}$precursors arise from IDP or CD4SP thymocytes (Tconv) with rates $f_{3}$ and $f_{4}$ Therefore, the IDP thymocyte pool follows the equation:

Structure A

$$
\frac{d[l D P]}{d t}=F_{\mathrm{eDP} \rightarrow \mathrm{DP}}-\left(d_{\mathrm{DP}}+f_{2}+f_{3}+f_{4}+f_{5}\right)[l D P]
$$

Structure B

$$
\frac{d[l D P]}{d t}=F_{\mathrm{eDP} \rightarrow \mathrm{IDP}}-\left(d_{\mathrm{DP}}+f_{2}+f_{5}\right)[l D P]
$$

Structure C

$$
\frac{d[l D P]}{d t}=F_{\mathrm{eDP} \rightarrow \mathrm{DP}}-\left(d_{\mathrm{DP}}+f_{2}+f_{3}+f_{5}\right)[l D P]
$$

Although the eDP-IDP model is able to recover from atrophy quite fast, we realized that a compensatory mechanism on proliferation actually gives a slightly faster recovery and better fit, especially for hypothesis 1 . This compensation was made as a 'logistic growth' term by using a dynamical number of divisions $N_{\text {div }}(t)$. We name $N_{\text {divinit }}$ the initial number of divisions of eDP thymocytes at steady state and $K a$ 'carrying capacity' of the thymus. $T(t)$ represents the total number of $T$ cells in the simulation, and $\alpha$ is scaled such that $N_{\text {div }}=N_{\text {div, init }}$ at steadystate.

$$
N_{\text {div }}(t)=N_{\text {div, init }} \alpha(1-T(t) / K)
$$

Death and output are the major driving mechanisms of SP thymocyte populations, which is compatible with an exponentially distributed residence time resulting from a single ODE. Proliferation, death, and thymic output are taken as constant rates named $p_{\mathrm{x}}, d_{\mathrm{x}}$, and $o_{\mathrm{x}}$, where $\mathrm{x}$ is the population name for SP thymocyte populations. $f_{6}$ and $f_{7}$ represent a constant differentiation rate between Treg cell precursors to mature Treg cells. 
structure A

$$
\begin{aligned}
\frac{d[\text { Tconv }]}{d t} & =f_{2}[l D P]+\left(p_{4}-d_{4}-o_{4}\right)[\text { Tconv }] \\
\frac{d[\text { CD } 25 \text { Prec }]}{d t} & =f_{3}[l D P]+\left(p_{\mathrm{H}}-d_{\mathrm{H}}-f_{7}-o_{\mathrm{H}}\right)[\text { CD } 25 \text { Prec }] \\
\frac{d[\text { Foxp } 3 \text { Prec }]}{d t} & =f_{4}[l D P]+\left(p_{\mathrm{S}}-d_{\mathrm{S}}-f_{6}-o_{\mathrm{S}}\right)[\text { Foxp } 3 \text { Prec }]
\end{aligned}
$$

structure B

$$
\begin{aligned}
\frac{d[\text { Tconv }]}{d t} & =f_{2}[\text { lDP }]+\left(p_{4}-d_{4}-o_{4}-f_{3}-f_{4}\right)[\text { Tconv }] \\
\frac{d[\text { CD25Prec }]}{d t} & =f_{3}[\text { tConv }]+\left(p_{\mathrm{H}}-d_{\mathrm{H}}-f_{7}-o_{\mathrm{H}}\right)[\text { CD } 25 \text { Prec }] \\
\frac{d[\text { Foxp } 3 \text { Prec }]}{d t} & =f_{4}[\text { tConv }]+\left(p_{\mathrm{S}}-d_{\mathrm{S}}-f_{6}-o_{\mathrm{S}}\right)[\text { Foxp } 3 \text { Prec }]
\end{aligned}
$$

structure C

$$
\begin{aligned}
\frac{d[\text { Tconv }]}{d t} & =f_{2}[l D P]+\left(p_{4}-d_{4}-o_{4}-f_{4}\right)[\text { Tconv }] \\
\frac{d[\text { CD } 25 \text { Prec }]}{d t} & =f_{3}[l D P]+\left(p_{\mathrm{H}}-d_{\mathrm{H}}-f_{7}-o_{\mathrm{H}}\right)[\text { CD } 25 \text { Prec }] \\
\frac{d[\text { Foxp } 3 \text { Prec }]}{d t} & =f_{4}[\text { tConv }]+\left(p_{\mathrm{S}}-d_{\mathrm{S}}-f_{6}-o_{\mathrm{S}}\right)[\text { Foxp } 3 \text { Prec }]
\end{aligned}
$$

And for all model structures,

$$
\begin{aligned}
\frac{d[\text { tTreg }]}{d t} & =f_{6}[\text { Foxp } 3 \text { Prec }]+f_{7}[\text { CD } 25 \text { Prec }]+\left(p_{\mathrm{R}}-d_{\mathrm{R}}-o_{\mathrm{R}}\right)[\text { tTreg }] \\
\frac{d[\text { tSP } 8]}{d t} & =f_{5}[\text { lDP }]+\left(p_{8}-d_{8}-o_{8}\right)[\text { tSP } 8]
\end{aligned}
$$

Parameter values: The parameter values for Tconv and CD8SP thymocyte dynamics were determined according to two constraints: (i) The proliferation rate is derived from the death and output rates to maintain experimental steady-state values, and (ii) the residence time of cells in the CD4SP and CD8SP thymocyte compartment was estimated to be 96 hours, which is calibrated as $1 /\left(o_{x}+d_{x}-p_{x}\right)$ in the model [55]. This suffices to determine the differentiation rate of IDP into CD4SP and CD8SP thymocytes, and to estimate the proliferation rate as a function of the death and output parameters. The remaining parameters (death and output of Tconv or CD8SP thymocytes, respectively) were completely insensitive, and the curves during atrophy were independent of their values (data not shown). Therefore, death and output rates were fixed with realistic values.

For the Treg cell precursors and mature Treg cell populations, there is no clear consensus on their residence time in the thymus, so only the steady state constraint was applied and the proliferation rate $p_{x}$ of each population is taken as a function of its death and output rates 
$d x$ and $o x$, which are also insensitive. The death rates $d x$ were estimated following two experimental constraints: (i) CD25+Foxp3- precursors die on average 3.67 times more than CD25-Foxp3 ${ }^{+}$precursors, as measured by Annexin $\mathrm{V}$ staining in Owen et al. [6], and (ii) mature tTreg cells die on average 1.9 times more than Tconv [56]. We assumed that CD25-Foxp $3^{+}$Treg cell precursors and mature Treg cells die with a similar strength.

Four main parameters are unknown: i) conversion rate from IDP thymocytes to CD25+Foxp3precursors, ii) conversion rate from IDP thymocytes to CD25-Foxp ${ }^{+}$precursors, iii) conversion rate from $\mathrm{CD}^{2} 5^{+}$Foxp $3^{-}$precursors to mature tTreg cells and iv) conversion rate from CD25-Foxp $3^{+}$precursors to mature tTreg cells. Similarly as for Tconv and CD8SP thymocytes, the death and output parameters were insensitive and could be fixed without impact. The list of parameters fixed in the system are summarized in Table 1.

\begin{tabular}{|c|c|c|c|}
\hline short name & parameter name & value & unit \\
\hline I & inflow eDPs & 452600 & cells/day \\
\hline $\mathrm{N}_{\text {div }}$ & eDPs divisions & 5.5341 & avg $\mathrm{nr}$ of divisions \\
\hline peDP & expansion rate eDPs & 4.5 & fraction / day \\
\hline$d_{\text {eDP }}$ & death rate eDPs & 0 & fraction / day \\
\hline$d_{D P}$ & death rate IDPs & 0.3441 & fraction / day \\
\hline$f_{2}$ & differentiation rate from DP to Tconv & 0.03905 & fraction / day \\
\hline$f_{5}$ & differentiation rate from DP to CD8SP & 0.012975 & fraction / day \\
\hline $\mathrm{p}_{4}$ & expansion rate Tconv & 0.03718 & fraction / day, derived \\
\hline $\mathrm{d}_{4}$ & death rate Tconv & 0.17819 & fraction / day, fixed \\
\hline $\mathrm{O}_{4}$ & output rate Tconv & 0.12 & fraction / day \\
\hline $\mathrm{p}_{8}$ & expansion rate CD8SP & 0.1456 & fraction / day, derived \\
\hline $\mathrm{d}_{8}$ & death rate CD8SP & 0.1066 & fraction / day, fixed \\
\hline O8 & output rate CD8SP & 0.3 & fraction / day, fixed \\
\hline $\mathrm{dH}_{\mathrm{H}}$ & death $\mathrm{CD}_{25^{+} \mathrm{Foxp} 3^{-}}$ & 1.2426 & fraction / day \\
\hline $\mathrm{p}_{\mathrm{H}}$ & expansion rate $\mathrm{CD}^{2} 5^{+}$Foxp $3^{-}$ & derived & fraction / day \\
\hline ds & death CD25-Foxp $3^{+}$ & 0.33857 & fraction / day \\
\hline ps & expansion rate CD25-Foxp3 ${ }^{+}$ & derived & fraction / day \\
\hline$d_{R}$ & death mature Treg cells & 0.33857 & fraction / day \\
\hline$p_{R}$ & expansion rate mature Treg cells & derived & fraction / day \\
\hline $\mathrm{OR}_{\mathrm{R}}$ & output rate mature Treg cells & 0.3485 & fraction / day, fixed \\
\hline$\alpha$ & logistic strength & derived & no unit \\
\hline
\end{tabular}

Table 1: Parameter values fixed before optimization. Proliferation rates are derived from other parameter values under steady-state constraint. The proliferation of Treg cell precursors and Treg cells will depend on the differentiation rates that are unknown and included in the fittings, therefore called 'derived'. 
Parameter optimization: The compatibility of different atrophy models (model structure and atrophy hypotheses) was assessed by parameter optimization ('fitting') using Stochastic Ranking Evolution Strategy algorithm [57] and by comparing simulations with the experimental dataset. The used cost function is the Residual Sum of Squares (RSS) after normalizing each population by its average value along all time-points, such that each population has a balanced contribution to the total cost. Best fitted parameter values are shown in Table 2, together with the RSS.

The AICc compares the quality/cost of models with different number of parameters. A minimal AICc value defines the model with the best fit and a minimal number of parameters. A difference of 2 in the AICc value is taken as significant improvement as a rule of thumb [58]. We assume a Gaussian distribution of noise in the $n=169$ data points, but with different strength per data point. The AICc formula is derived from the likelihood $L$ of the simulated points $x_{\theta, j}$ with the best parameter set $\theta$ as following, with $k$ parameters, $\hat{\sigma}_{j}$ and $\hat{\mu}_{j}$ the estimated variance and mean of each data point $j$ :

$$
\begin{gathered}
L_{\theta \mid \text { data }}=\prod_{j=1}^{n} \frac{1}{2 \pi \hat{\sigma}_{j}} \exp \left(-\frac{\left(x_{\theta, j}-\hat{\mu}_{j}\right)^{2}}{2 \hat{\sigma}_{j}}\right) \\
A I C_{c}=-2 \ln \left(L_{\theta \mid \text { data }}\right)+2 \frac{k n}{n-k-1} \\
=C+\sum_{j=1}^{n} \frac{\left(x_{\theta, j}-\hat{\mu}_{j}\right)^{2}}{\hat{\sigma}_{j}}+2 \frac{k n}{n-k-1}
\end{gathered}
$$

C represents a constant that is neglected when comparing the difference of AICc values between models, and is ignored in the calculation.

The experimental dataset, details of modeling hypotheses and parameter estimation results for all model structures are given in Supporting Information 'Mathematical Model'. The simulation code, written in $\mathrm{C}++$ using the Moonfit framework [59], was deposited at https://gitlab.com/Moonfit/Balthyse. 


\begin{tabular}{|c|c|c|c|c|c|c|}
\hline parameter & $\begin{array}{c}\text { hypothesis } \\
1\end{array}$ & $\begin{array}{c}\text { hypothesis } \\
2\end{array}$ & $\begin{array}{c}\text { hypothesis } \\
3\end{array}$ & $\begin{array}{c}\text { hypothesis } \\
4\end{array}$ & $\min$ & $\max$ \\
\hline factor F peak (days) & 8.493 & 8.586 & 8.492 & 8.95 & 5 & 15 \\
\hline factor F width (days) & 1.937 & 1.841 & 1.07 & 1.505 & 0.25 & 10 \\
\hline diff. DP (or CD4SP) to CD25 $5^{+}$Foxp3 $3^{-}$ & 0.0116 & 0.013 & 0.01071 & 0.0002 & 0.0002 & 0.1 \\
\hline diff. DP (or CD4SP) to CD25-Foxp3+ & 0.0108 & 0.0097 & 0.0054 & 0.00116 & 0.0002 & 0.1 \\
\hline diff. CD25 $5^{+}$Foxp $3^{-}$to mature Treg cells & 0.808 & 1.035 & 0.634 & 0.017 & 0.01 & 2 \\
\hline diff. CD25-Foxp3 ${ }^{+}$to mature Treg cells & 0.312 & 0.236 & 0.011 & 0.01 & 0.01 & 2 \\
\hline added death rate eDPs & 2.413 & 1.722 & 6.362 & 2.046 & $+0.01 / d$ & $+20 / d$ \\
\hline multiplied to death rate IDPs & 3.929 & 4.995 & 4.791 & 7.742 & $x 0.01$ & $x 20$ \\
\hline added output rate Tconvs & - & - & 0.256 & 0.153 & $+0.01 / d$ & $+20 / d$ \\
\hline added output rate mature Treg cells & - & - & 0.256 & 0.393 & $+0.01 / d$ & $+20 / d$ \\
\hline added output rate CD25-Foxp3 ${ }^{+}$ & - & - & 0.256 & 0.393 & $+0.01 / d$ & $+20 / d$ \\
\hline added output rate CD25+Foxp3- & - & - & 0.256 & 0.393 & $+0.01 / d$ & $+20 / d$ \\
\hline added output rate CD8SP & - & - & 0.256 & - & $+0.01 / d$ & $+20 / d$ \\
\hline multiplied diff. into CD25-Foxp3 ${ }^{+}$ & - & 1.00006 & - & - & $x 1$ & $x 20$ \\
\hline carrying capacity thymus & 409 & 799.7 & 799.8 & 312.8 & 150 & 800 \\
\hline Cost (norm RSS) & 13 & 12.8 & 11 & 10 & & \\
\hline Number of parameters & 9 & 10 & 10 & 11 & & \\
\hline AICc index & 259.3 & 256.1 & 219.9 & 194.4 & & \\
\hline
\end{tabular}

Table 2: Parameter boundaries, best parameter sets and RSS costs obtained during the fitting of each hypothesis for model structure B and corresponding to the curves shown in Figure 6.

\section{TCR profiling}

For the TCR repertoire analysis, Treg cells and Tconv were sorted from thymi of control or

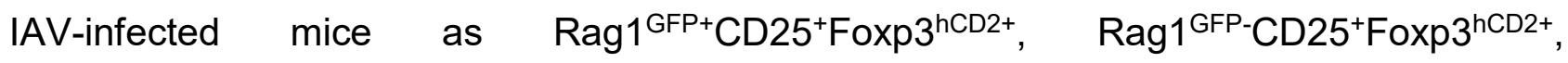
Rag $1{ }^{\text {GFP+ }}$ CD25-Foxp3 ${ }^{\text {hCD2- }}$, and Rag1 ${ }^{\text {GFP-CD25-Foxp3 }}{ }^{\text {hCD2- }}$ CD4SP thymocyte subsets. A 5'RACE cDNA synthesis, followed by a PCR employing a mTCR $\beta$ constant region reverse primer and a UPM (universal primer mix), was performed. In brief, RNA extraction from sorted cells was performed by using RNeasy Plus Micro kit (Qiagen). The cDNA was generated by employing ingredients from the TAKARA cDNA Synthesis Kit (TAKARA Bio Europe), based on the manufacturer's protocol. A mixture from $1 \mu \mathrm{l} 12 \mu \mathrm{M}$ OligodT and $4.5 \mu \mathrm{l}$ RNA was incubated for $3 \mathrm{~min}$ at $72^{\circ} \mathrm{C}$, followed by $2 \mathrm{~min}$ at $42^{\circ} \mathrm{C}$ and placed on ice immediately. Directly, $4.5 \mu \mathrm{l}$ RACE-Mastermix (1X First-Strand-buffer, $2.5 \mathrm{mM}$ DTT, $1 \mathrm{mM}$ dNTP mix, $0.6 \mu \mathrm{M}$ SMARTer IIA Oligonucleotide, $10 \mathrm{U}$ RNase Inhibitor and $50 \mathrm{U}$ SMARTScribe Reverse Transcriptase) was added, and the PCR program was continued for 
$90 \mathrm{~min}$ at $42^{\circ} \mathrm{C}$ and $10 \mathrm{~min}$ at $72^{\circ} \mathrm{C}$. Subsequently, the DNA-amplicons for Illumina sequencing were generated by using Advantage 2 PCR Kit (TAKARA Bio Europe), employing a mTCR $\beta$ reverse primer and a Universal primer mix (UPM). The fully generated cDNA $(10 \mu \mathrm{l})$ was processed. After agarose-electrophoresis, the DNA was purified using the Qiaquick Gel Extraction Kit (Qiagen). The purified product of each sample was indexed by an indexing-PCR with Nextera-Primers. Finally, a pool of the samples was sequenced, employing Illumina MiSeq v2 Reagent Kit (Illumina). For the analysis of TCR sequencing data, samples were aligned to the international immunogenetics information system (IMGT) database from 22.05.2018, using MiXCR software [60]. Then, the TCR diversity analysis (inverse Simpson's index) was performed by employing VDJTools [61]. The analyses were done to the complete data sets, and to thymus samples down-sampled to 20,000 counts (i.e. productive reads) after assessment of read-numbers in all samples. Sequencing data were deposited at NCBI GEO with accession number GSE139499.

\section{Statistical analysis}

The GraphPad Prism software v7.0 was used to perform all statistical analyses. Data are presented as mean \pm or + standard deviation (SD). For comparison of unmatched groups, the two-tailed Mann-Whitney test was performed, and the p-values were calculated with the long-rank test (Mantel-Cox). A p-value below 0.05 was considered as significant; ${ }^{*} p<0.05 ;{ }^{* *} p<0.01 ;{ }^{* *} p<0.001 ;{ }^{* * *} p<0.0001$; ns (not significant).

Acknowledgements: We thank Lothar Gröbe for cell sorting, Maria Ebel for technical support and Marcus Gereke. This work was supported by PhD scholarship programme no. 57129429 of the German Academic Exchange Service (DAAD) (to Y.E.). P.A.R. was supported by the Human Frontier Science Program (RGP0033/2015).

Conflicts of interest: The authors declare no commercial or financial conflict of interest. 


\section{REFERENCES}

1 Sakaguchi, S., Mikami, N., Wing, J. B., Tanaka, A., Ichiyama, K. and Ohkura, N., Regulatory T Cells and Human Disease. Annu Rev Immunol 2020. 38: 541-566.

2 Klein, L., Robey, E. A. and Hsieh, C. S., Central CD4 ${ }^{+}$T cell tolerance: deletion versus regulatory T cell differentiation. Nat Rev Immunol 2019. 19: 7-18.

3 Lio, C. W. and Hsieh, C. S., A two-step process for thymic regulatory T cell development. Immunity 2008. 28: 100111.

4 Tai, X., Erman, B., Alag, A., Mu, J., Kimura, M., Katz, G., Guinter, T., McCaughtry, T., Etzensperger, R., Feigenbaum, L., Singer, D. S. and Singer, A., Foxp3 transcription factor is proapoptotic and lethal to developing regulatory T cells unless counterbalanced by cytokine survival signals. Immunity 2013. 38: 1116-1128.

5 Marshall, D., Sinclair, C., Tung, S. and Seddon, B., Differential requirement for IL-2 and IL-15 during bifurcated development of thymic regulatory T cells. J Immunol 2014. 193: 5525-5533.

6 Owen, D. L., Mahmud, S. A., Sjaastad, L. E., Williams, J. B., Spanier, J. A., Simeonov, D. R., Ruscher, R., Huang, W., Proekt, I., Miller, C. N., Hekim, C., Jeschke, J. C., Aggarwal, P., Broeckel, U., LaRue, R. S., Henzler, C. M., Alegre, M. L., Anderson, M. S., August, A., Marson, A., Zheng, Y., Williams, C. B. and Farrar, M. A., Thymic regulatory T cells arise via two distinct developmental programs. Nat Immunol 2019. 20: 195-205.

7 Onodera, T., Taniguchi, T., Tsuda, T., Yoshihara, K., Shimizu, S., Sato, M., Awaya, A. and Hayashi, T., Thymic atrophy in type 2 reovirus infected mice: immunosuppression and effects of thymic hormone. Thymic atrophy caused by reo-2. Thymus 1991. 18: 95-109.

8 Savino, W., The thymus is a common target organ in infectious diseases. PLoS Pathog 2006. 2: e62.

9 Majumdar, S. and Nandi, D., Thymic Atrophy: Experimental Studies and Therapeutic Interventions. Scand J Immunol 2018. 87: 4-14.

10 Cepeda, S. and Griffith, A. V., Thymic stromal cells: Roles in atrophy and age-associated dysfunction of the thymus. Exp Gerontol 2018. 105: 113-117.

11 Venables, T., Griffith, A. V., DeAraujo, A. and Petrie, H. T., Dynamic changes in epithelial cell morphology control thymic organ size during atrophy and regeneration. Nat Commun 2019. 10: 4402.

12 Alawam, A. S., Anderson, G. and Lucas, B., Generation and Regeneration of Thymic Epithelial Cells. Front Immunol 2020. 11: 858.

13 Dooley, J. and Liston, A., Molecular control over thymic involution: from cytokines and microRNA to aging and adipose tissue. Eur J Immunol 2012. 42: 1073-1079.

14 Anz, D., Thaler, R., Stephan, N., Waibler, Z., Trauscheid, M. J., Scholz, C., Kalinke, U., Barchet, W., Endres, S. and Bourquin, C., Activation of melanoma differentiation-associated gene 5 causes rapid involution of the thymus. J Immunol 2009. 182: 6044-6050.

15 Papadopoulou, A. S., Dooley, J., Linterman, M. A., Pierson, W., Ucar, O., Kyewski, B., Zuklys, S., Hollander, G. A., Matthys, P., Gray, D. H., De Strooper, B. and Liston, A., The thymic epithelial microRNA network elevates the threshold for infection-associated thymic involution via miR-29a mediated suppression of the IFN-alpha receptor. Nat Immunol 2011. 13: 181-187.

16 Mehr, R., Globerson, A. and Perelson, A. S., Modeling positive and negative selection and differentiation processes in the thymus. $J$ Theor Biol 1995. 175: 103-126.

17 Thomas-Vaslin, V., Altes, H. K., de Boer, R. J. and Klatzmann, D., Comprehensive assessment and mathematical modeling of T cell population dynamics and homeostasis. J Immunol 2008. 180: 2240-2250.

18 Moleriu, R. D., Zaharie, D., Moatar-Moleriu, L. C., Gruia, A. T., Mic, A. A. and Mic, F. A., Insights into the mechanisms of thymus involution and regeneration by modeling the glucocorticoid-induced perturbation of thymocyte populations dynamics. J Theor Biol 2014. 348: 80-99.

19 Sinclair, C., Bains, I., Yates, A. J. and Seddon, B., Asymmetric thymocyte death underlies the CD4:CD8 T-cell ratio in the adaptive immune system. Proc Natl Acad Sci U S A 2013. 110: E2905-2914.

20 Tumpey, T. M. and Belser, J. A., Resurrected pandemic influenza viruses. Annu Rev Microbiol 2009. 63: $79-98$.

21 Vogel, A. B., Haasbach, E., Reiling, S. J., Droebner, K., Klingel, K. and Planz, O., Highly pathogenic influenza virus infection of the thymus interferes with T lymphocyte development. J Immunol 2010. 185: 4824-4834.

22 Liu, B., Zhang, X., Deng, W., Liu, J., Li, H., Wen, M., Bao, L., Qu, J., Liu, Y., Li, F., An, Y., Qin, C., Cao, B. and Wang, C., Severe influenza $A(\mathrm{H} 1 \mathrm{~N} 1)$ pdm09 infection induces thymic atrophy through activating innate $\mathrm{CD} 8^{+} \mathrm{CD} 44^{\mathrm{hi}}$ T cells by upregulating IFN-gamma. Cell Death Dis 2014. 5: e1440.

23 Duan, X., Lu, J., Zhou, K., Wang, J., Wu, J., Gao, G. F. and Fang, M., NK-cells are involved in thymic atrophy induced by influenza A virus infection. J Gen Virol 2015. 96: 3223-3235. 
24 Thiault, N., Darrigues, J., Adoue, V., Gros, M., Binet, B., Perals, C., Leobon, B., Fazilleau, N., Joffre, O. P., Robey, E. A., van Meerwijk, J. P. and Romagnoli, P., Peripheral regulatory T lymphocytes recirculating to the thymus suppress the development of their precursors. Nat Immunol 2015. 16: 628-634.

25 Cowan, J. E., McCarthy, N. I. and Anderson, G., CCR7 Controls Thymus Recirculation, but Not Production and Emigration, of Foxp3 ${ }^{+}$T Cells. Cell Rep 2016. 14: 1041-1048.

26 Cowan, J. E., Baik, S., McCarthy, N. I., Parnell, S. M., White, A. J., Jenkinson, W. E. and Anderson, G., Aire controls the recirculation of murine Foxp3 ${ }^{+}$regulatory T-cells back to the thymus. Eur J Immunol 2018. 48: 844-854.

27 Herppich, S., Toker, A., Pietzsch, B., Kitagawa, Y., Ohkura, N., Miyao, T., Floess, S., Hori, S., Sakaguchi, S. and Huehn, J., Dynamic Imprinting of the Treg Cell-Specific Epigenetic Signature in Developing Thymic Regulatory $T$ Cells. Front Immunol 2019. 10: 2382.

28 Nikolouli, E., Elfaki, Y., Herppich, S., Schelmbauer, C., Delacher, M., Falk, C., Mufazalov, I. A., Waisman, A., Feuerer, M. and Huehn, J., Recirculating IL-1R2 ${ }^{+}$Tregs fine-tune intrathymic Treg development under inflammatory conditions. Cell Mol Immunol 2020.

29 Peligero-Cruz, C., Givony, T., Sebe-Pedros, A., Dobes, J., Kadouri, N., Nevo, S., Roncato, F., Alon, R., Goldfarb, Y. and Abramson, J., IL18 signaling promotes homing of mature Tregs into the thymus. Elife 2020. 9.

30 Kuwata, N., Igarashi, H., Ohmura, T., Aizawa, S. and Sakaguchi, N., Cutting edge: absence of expression of RAG1 in peritoneal B-1 cells detected by knocking into RAG1 locus with green fluorescent protein gene. J Immunol 1999. 163: 6355-6359.

31 Burchill, M. A., Yang, J., Vang, K. B., Moon, J. J., Chu, H. H., Lio, C. W., Vegoe, A. L., Hsieh, C. S., Jenkins, M. K. and Farrar, M. A., Linked T cell receptor and cytokine signaling govern the development of the regulatory $T$ cell repertoire. Immunity 2008. 28: 112-121.

32 Hwang, S., Song, K. D., Lesourne, R., Lee, J., Pinkhasov, J., Li, L., El-Khoury, D. and Love, P. E., Reduced TCR signaling potential impairs negative selection but does not result in autoimmune disease. J Exp Med 2012. 209: 17811795.

33 Oh, J., Wang, W., Thomas, R. and Su, D. M., Capacity of tTreg generation is not impaired in the atrophied thymus. PLOS Biol 2017. 15: e2003352.

34 Lyszkiewicz, M., Winter, S. J., Witzlau, K., Fohse, L., Brownlie, R., Puchalka, J., Verheyden, N. A., KunzeSchumacher, H., Imelmann, E., Blume, J., Raha, S., Sekiya, T., Yoshimura, A., Frueh, J. T., Ullrich, E., Huehn, J., Weiss, S., Gutierrez, M. G., Prinz, I., Zamoyska, R., Zietara, N. and Krueger, A., miR-181a/b-1 controls thymic selection of Treg cells and tunes their suppressive capacity. PLoS Biol 2019. 17: e2006716.

35 Azzam, H. S., DeJarnette, J. B., Huang, K., Emmons, R., Park, C. S., Sommers, C. L., El-Khoury, D., Shores, E. W. and Love, P. E., Fine tuning of TCR signaling by CD5. J Immunol 2001. 166: 5464-5472.

36 Moran, A. E., Holzapfel, K. L., Xing, Y., Cunningham, N. R., Maltzman, J. S., Punt, J. and Hogquist, K. A., T cell receptor signal strength in Treg and iNKT cell development demonstrated by a novel fluorescent reporter mouse. $J$ Exp Med 2011. 208: 1279-1289.

37 Toomer, K. H. and Malek, T. R., Cytokine Signaling in the Development and Homeostasis of Regulatory T cells. Cold Spring Harb Perspect Biol 2018. 10.

38 Liu, Y., Zhang, P., Li, J., Kulkarni, A. B., Perruche, S. and Chen, W., A critical function for TGF-beta signaling in the development of natural $\mathrm{CD}^{+}{ }^{+} \mathrm{CD} 25^{+} \mathrm{Foxp}^{+}$regulatory T cells. Nat Immunol 2008. 9: 632-640.

39 Coder, B. D., Wang, H., Ruan, L. and Su, D. M., Thymic involution perturbs negative selection leading to autoreactive T cells that induce chronic inflammation. J Immunol 2015. 194: 5825-5837.

40 Zheng, Y., Josefowicz, S., Chaudhry, A., Peng, X. P., Forbush, K. and Rudensky, A. Y., Role of conserved noncoding DNA elements in the Foxp3 gene in regulatory T-cell fate. Nature 2010. 463: 808-812.

41 Ouyang, W., Beckett, O., Ma, Q. and Li, M. O., Transforming growth factor-beta signaling curbs thymic negative selection promoting regulatory T cell development. Immunity 2010. 32: 642-653.

42 Konkel, J. E., Jin, W., Abbatiello, B., Grainger, J. R. and Chen, W., Thymocyte apoptosis drives the intrathymic generation of regulatory T cells. Proc Natl Acad Sci U S A 2014. 111: E465-473.

43 Schuster, M., Plaza-Sirvent, C., Matthies, A. M., Heise, U., Jeron, A., Bruder, D., Visekruna, A., Huehn, J. and Schmitz, I., c-REL and IkappaBNS Govern Common and Independent Steps of Regulatory T Cell Development from Novel CD122-Expressing Pre-Precursors. J Immunol 2017. 199: 920-930.

44 Khailaie, S., Robert, P. A., Toker, A., Huehn, J. and Meyer-Hermann, M., A signal integration model of thymic selection and natural regulatory T cell commitment. J Immunol 2014. 193: 5983-5996.

45 Aschenbrenner, K., D'Cruz, L. M., Vollmann, E. H., Hinterberger, M., Emmerich, J., Swee, L. K., Rolink, A. and Klein, L., Selection of Foxp3 ${ }^{+}$regulatory T cells specific for self antigen expressed and presented by Aire ${ }^{+}$medullary thymic epithelial cells. Nat Immunol 2007. 8: 351-358. 
46 Proietto, A. I., van Dommelen, S., Zhou, P., Rizzitelli, A., D'Amico, A., Steptoe, R. J., Naik, S. H., Lahoud, M. H., Liu, Y., Zheng, P., Shortman, K. and Wu, L., Dendritic cells in the thymus contribute to T-regulatory cell induction. Proc Natl Acad Sci U S A 2008. 105: 19869-19874.

47 Spence, P. J. and Green, E. A., Foxp3 ${ }^{+}$regulatory T cells promiscuously accept thymic signals critical for their development. Proc Natl Acad Sci U S A 2008. 105: 973-978.

48 Coquet, J. M., Ribot, J. C., Babala, N., Middendorp, S., van der Horst, G., Xiao, Y., Neves, J. F., Fonseca-Pereira, D., Jacobs, H., Pennington, D. J., Silva-Santos, B. and Borst, J., Epithelial and dendritic cells in the thymic medulla promote CD4+Foxp3 ${ }^{+}$regulatory T cell development via the CD27-CD70 pathway. J Exp Med 2013. 210: 715-728.

49 Garg, G., Nikolouli, E., Hardtke-Wolenski, M., Toker, A., Ohkura, N., Beckstette, M., Miyao, T., Geffers, R., Floess, S., Gerdes, N., Lutgens, E., Osterloh, A., Hori, S., Sakaguchi, S., Jaeckel, E. and Huehn, J., Unique properties of thymic antigen-presenting cells promote epigenetic imprinting of alloantigen-specific regulatory $\mathrm{T}$ cells. Oncotarget 2017. 8: 35542-35557.

50 Xia, J., Wang, H., Guo, J., Zhang, Z., Coder, B. and Su, D. M., Age-Related Disruption of Steady-State Thymic Medulla Provokes Autoimmune Phenotype via Perturbing Negative Selection. Aging Dis 2012. 3: 248-259.

51 Carrio, R. and Lopez, D. M., Insights into thymic involution in tumor-bearing mice. Immunol Res 2013. 57: $106-114$.

52 Sun, S., Ji, H., Feng, Y., Kang, Y., Yu, J. and Liu, A., A novel mechanism of tumor-induced thymic atrophy in mice bearing H22 hepatocellular carcinoma. Cancer Manag Res 2018. 10: 417-424.

53 Miyao, T., Floess, S., Setoguchi, R., Luche, H., Fehling, H. J., Waldmann, H., Huehn, J. and Hori, S., Plasticity of Foxp $3^{+} \mathrm{T}$ cells reflects promiscuous Foxp3 expression in conventional T cells but not reprogramming of regulatory $\mathrm{T}$ cells. Immunity 2012. 36: 262-275.

54 Cossarizza, A., Chang, H. D., Radbruch, A., Acs, A., Adam, D., Adam-Klages, S., Agace, W. W., Aghaeepour, N., Akdis, M., Allez, M., Almeida, L. N., Alvisi, G., Anderson, [...], Engelhardt, B., Erdei, A., Guidelines for the use of flow cytometry and cell sorting in immunological studies (second edition). Eur J Immunol 2019. 49: 1457-1973.

55 Stritesky, G. L., Xing, Y., Erickson, J. R., Kalekar, L. A., Wang, X., Mueller, D. L., Jameson, S. C. and Hogquist, K. A., Murine thymic selection quantified using a unique method to capture deleted T cells. Proc Natl Acad Sci U S A 2013. 110: 4679-4684.

56 Plaza-Sirvent, C., Schuster, M., Neumann, Y., Heise, U., Pils, M. C., Schulze-Osthoff, K. and Schmitz, I., c-FLIP Expression in Foxp3-Expressing Cells Is Essential for Survival of Regulatory T Cells and Prevention of Autoimmunity. Cell Rep 2017. 18: 12-22.

$57 \mathbf{J i}, \mathbf{X}$. and $\mathbf{X u}, \mathbf{Y}$., libSRES: a C library for stochastic ranking evolution strategy for parameter estimation. Bioinformatics 2006. 22: 124-126.

58 Burnham, K. D. and Anderson, D. R., Multimodel Inference: Understanding AIC and BIC in Model Selection. Sociological Methods \& Research 2004. 33: 261-304.

59 Robert, P. A., Jönsson, H. and Meyer-Hermann, M., MoonFit, a minimal interface for fitting ODE dynamical models, bridging simulation by experimentalists and customization by $\mathrm{C}++$ programmers. bioRxiv 2018. 281188.

60 Bolotin, D. A., Poslavsky, S., Mitrophanov, I., Shugay, M., Mamedov, I. Z., Putintseva, E. V. and Chudakov, D. M., MiXCR: software for comprehensive adaptive immunity profiling. Nat Methods 2015. 12: 380-381.

61 Shugay, M., Bagaev, D. V., Turchaninova, M. A., Bolotin, D. A., Britanova, O. V., Putintseva, E. V., Pogorelyy, M. V., Nazarov, V. I., Zvyagin, I. V., Kirgizova, V. I., Kirgizov, K. I., Skorobogatova, E. V. and Chudakov, D. M., VDJtools: Unifying Post-analysis of T Cell Receptor Repertoires. PLoS Comput Biol 2015. 11: e1004503. 